\title{
Conocimiento y uso de los servicios de la biblioteca de la Universidad del País Vasco/Euskal Herriko Unibertsitatea. Diferencias entre usuarios y áreas de conocimiento
}

\author{
Luis-María FERNÁNDEZ MARTÍNEZ \\ Biblioteca de la Universidad del País Vasco/Euskal Herriko Unibertsitatea \\ luismaria.fernandez@ehu.es
}

Recibido: 20/02/2011

Aceptado: 18/03/2011

\section{RESUMEN}

Ante el nuevo contexto creado con la implantación del EEES y el sistema de ECTS, en 2009 se realizó una encuesta en la Universidad del País Vasco/Euskal Herriko Unibertsitatea dirigida al alumnado y al personal docente e investigador (PDI). En ella se incorporaron cuestiones relativas a los servicios ofrecidos por la Biblioteca, tanto presenciales como no presenciales, a fin de establecer el grado de conocimiento y uso de los mismos. El estudio muestra las diferencias detectadas tanto entre el alumnado y el PDI así como entre las diferentes áreas de conocimiento. Palabras-clave: Universidad del País Vasco/Euskal Herriko Unibertsitatea, Biblioteca universitaria, Servicios bibliotecarios, Estudio de usuarios.

Knowledge and use of the University of the Basque Country Library services. Users and disciplines differences

\begin{abstract}
In face of the new context created by EHEA and ECTS, in 2009 was made a survey to be aimed at students and teaching and research staff of the University of the Basque Country. Questions about the University Library services were included to set the knowledge and use of these services. The survey shows the differences detected between both groups of users and between disciplines.

Key words: University of the Basque Country, University library, Library services, Users survey.
\end{abstract}




\section{INTRODUCCIÓN}

El desarrollo del Espacio Europeo de Educación Superior (EEES) y la implantación del European Credits Transfer System (ECTS) crean el nuevo contexto de la Educación Superior. En dicho contexto se produce un cambio en la orientación de la misma al considerarse que "el esfuerzo del estudiante es más importante que el esfuerzo del profesor, ya que el fin último de la educación universitaria es desarrollar el autoaprendizaje y el pensamiento independiente del estudiante" (Lavigne, 2006).

Para ello "el profesor ha de tender a reemplazar su función de emisor de información por la función de tutor del proceso de aprendizaje (...)" y "esto no hará más cómodo el trabajo de los profesores: más bien al contrario, ya que se les exigirá una mayor competencia pedagógica y un mayor grado de motivación" (Bricall, 2000).

Por todo ello, este nuevo modelo requiere un mayor conocimiento y uso de los diferentes servicios de la biblioteca que reforzará su papel. En palabras de Orera (2007), "sin duda alguna, el nuevo método de aprendizaje que supone la implantación del EEES, contribuirá a lograr una mayor integración y peso de la biblioteca en las universidades, al basarse en una mayor participación del estudiante en el proceso de aprendizaje y requerir más utilización de recursos y servicios bibliográficos".

Dentro de las bibliotecas podemos distinguir tres componentes funcionales básicos: la colección, los medios de acceso a la colección, y la ayuda en el acceso y uso de la colección (Bailin y Grafstein, 2005). Con la incorporación de las tecnologías de la información y las comunicaciones (TICs) en estos tres componentes se observa cómo se han generado e incorporado nuevos productos que requieren, en ocasiones, nuevas formas y técnicas en la transmisión de la información e incluso traen consigo un cambio en la gestión de los recursos de la biblioteca universitaria.

\section{ESTADO DE LA CUESTIÓN}

Las bibliotecas siempre han necesitado saber cuál es el grado de conocimiento y uso de los diferentes servicios que ofrecen a sus usuarios. Ese factor de análisis de la adecuación de los servicios a la consecución de los fines de la biblioteca ha favorecido que estos estudios se hayan realizado, de uno u otro modo, desde los primeros desarrollos bibliotecarios. Sin embargo, la investigación sistemática sobre los usuarios de las bibliotecas es para Sridhar (1988) un logro del siglo XX, con especial impulso a partir de los años 40. En su obra, Sridhar recopila una variada y completa bibliografía que pone de manifiesto ese desarrollo y ofrece una amplia visión del mismo.

Centrándonos en los estudios realizados en el ámbito de las bibliotecas universitarias, podemos destacar las obras de Kent (1979), Trochim, Miller y Trochim (1985) y la de Harrel (1988). En ellas, además de plasmarse los resultados de los estudios, se recogen unas bibliografías que dan muestra de la creciente importancia de este campo de investigación.

Las bibliotecas universitarias españolas no se han sustraído a este desarrollo y también han llevado a cabo estudios sobre la materia entre los que destacamos los 
realizados en la Universidad de Murcia por Gómez Hernández (1996), en la Universidad de la Coruña por Perea Vega (2002) y en la Universidad Complutense por García Ochoa, Crego Castaño y Gimeno Perelló (1993) y por Arias Coello y Simón Martín (2008).

Todos ellos ofrecen, al igual que en las obras mencionadas con anterioridad, una rica información bibliográfica.

Sin embargo, ha habido un elemento tecnológico cuya aparición e implantación ha resultado de vital importancia y trascendencia en el mundo de las bibliotecas en las últimas décadas del siglo XX. Se trata de la aparición, en un primer momento, de los nuevos soportes de la información (CD-ROM, etc.) y, posteriormente, de Internet y sus aplicaciones con el desarrollo e implantación de nuevas tecnologías de la información y comunicación (TICs).

La incorporación de las nuevas tecnologías ha traído consigo cambios importantes en las bibliotecas universitarias, tanto en lo referente a sus colecciones, como a los medios de acceso y uso de dichas colecciones. Estos cambios se han plasmado en la modificación en los hábitos de los usuarios respecto a la biblioteca y en las formas de acceso y uso de los diferentes servicios de las bibliotecas como expuso Martell (2007), quien también recogió cómo han llegado a afectar incluso a la asistencia personal del usuario a la biblioteca universitaria (Martell, 2008).

Por ello, estas nuevas tecnologías se incorporan a los diferentes estudios realizados sobre los usuarios ${ }^{1} \mathrm{y}$, lo que es más importante, al aumentar los servicios ofrecidos con ellas en muchas ocasiones ha sido objeto de estudios individualizados. En el ámbito de bibliotecas universitarias españolas señalamos los estudios de Suarez Balseiro (2001), Borrego (2007) y Rodríguez Bravo (2008).

Una recopilación de trabajos sobre estos servicios se puede consultar en la obra de Tenopir (2003). Así mismo, tenemos una muestra de cómo sigue siendo objeto de estudio en la obra de Brown y Swan (2007).

Partiendo de esta situación, se plantea la realización de una investigación sobre el conocimiento y uso de los servicios ofrecidos por la biblioteca a la comunidad universitaria. Un estudio en el que, si bien se reúnen aspectos que otros trabajos han planteado de forma individual, contempla a la biblioteca universitaria como un todo, sin disgregarla de una realidad en la que se configura como biblioteca híbrida. En esta nueva configuración, en la que la biblioteca tradicional incorpora características de la biblioteca digital, la biblioteca universitaria ofrece diferentes servicios con distintas formas de acceso a los mismos.

En este estudio las TICs dejan de ser consideradas como un fin en sí mismas y pasan a ser contempladas por su carácter instrumental, como herramientas en manos de la Biblioteca. A partir de ese cambio de punto de vista, la investigación ofrece una nueva perspectiva y pone su énfasis en el estudio detallado de los servicios que ofrece la biblioteca universitaria como una característica esencial de su misión. Una perspectiva y objetivos poco frecuentes, como señala Ramos Simón (2009): “participamos de la

\footnotetext{
${ }^{1}$ Arias Coello y Simón Martin incluyeron en su estudio de forma genérica la conexión a Internet y el acceso a los recursos electrónicos.
} 
sorpresa de Patalano cuando subraya que si bien la idea de servicio resulta presente en prácticamente todas las bibliotecas y estudios sobre la misión de las bibliotecas, se presta una atención escasísima a estudiar tales servicios. Por citar un dato representativo, sólo el 0,01\% de los trabajos indizados en Library and Information Science Abstracts (Lisa) se ocupa del aspecto referido al servicio de las bibliotecas".

Por otro lado, ante el nuevo modelo de enseñanza que se implanta con el Espacio Europeo de Enseñanza Superior en el que cobra protagonismo el proceso de aprendizaje del alumno, se analizan las diferencias que se muestran en dicho conocimiento y uso entre el alumnado y el PDI.

Además el estudio se hace teniendo en cuenta las distintas áreas de conocimiento, con lo que los resultados obtenidos detectan las diferencias de comportamiento de las mismas (Hiller, 2002).

\section{METODOLOGÍA}

Entre el 15 de mayo y el 28 de junio de 2009 se realizó una encuesta entre el alumnado y el PDI de la Universidad del País Vasco/Euskal Herriko Unibertsitatea (UPV/EHU en adelante) como parte de un amplio estudio ${ }^{2}$. Dentro de la encuesta se incorporaron cuestiones relacionadas con el conocimiento y uso de los servicios ofrecidos por la biblioteca. Estos servicios se agruparon en presenciales y no presenciales.

La encuesta se confeccionó utilizando el servicio en línea "Encuesta Fácil" (www.encuestafacil.com), facilitado por la propia UPV/EHU. Para fomentar la participación en la misma se solicitó la colaboración mediante el envío de un correo electrónico en el que se incorporó el enlace a la dirección url de la encuesta correspondiente a cada grupo de usuario. En el caso del alumnado se utilizó su lista de distribución de la universidad mientras que al PDI se le envió directamente a sus direcciones de correo electrónico que tuvieron que ser recopiladas.

En total se recibieron 1390 respuestas del alumnado y 516 del PDI. Aplicando lo expuesto sobre tamaños muestrales por Hernández Blázquez (2001) los resultados ofrecen un grado de confianza del $95 \%$, con un margen de error de $\pm 3 \%$.

\section{CONOCIMIENTO Y USO DE SERVICIOS PRESENCIALES}

A continuación analizaremos los resultados correspondientes al conocimiento y uso de los servicios presenciales ${ }^{3}$ de la Biblioteca de la UPV/EHU, comparando ambos estamentos.

\footnotetext{
${ }^{2}$ FERNANDEZ MARTINEZ, Luis Ma . La Biblioteca de la Universidad del País Vasco ante la convergencia europea: conocimiento y uso de las TICS. Tesis inédita. Universidad de Zaragoza, Facultad de Filosofía y Letras, Departamento de Ciencias de la Documentación e Historia de la Ciencia, 2010.

${ }^{3}$ Los servicios presenciales identificados fueron: Consultar fondos bibliográficos en papel, libros; Consultar fondos bibliográficos en papel, revistas; Consultar bases de datos en monopuesto; Consultar
} 
A la vista del siguiente gráfico, se constata que el conocimiento y uso de estos servicios es mayor entre el PDI que entre el alumnado. Esta situación se invierte en el caso del trabajo con materiales propios y en el uso de los ordenadores de la Biblioteca (el PDI contaría con acceso a espacios y equipos similares fuera de la Biblioteca).

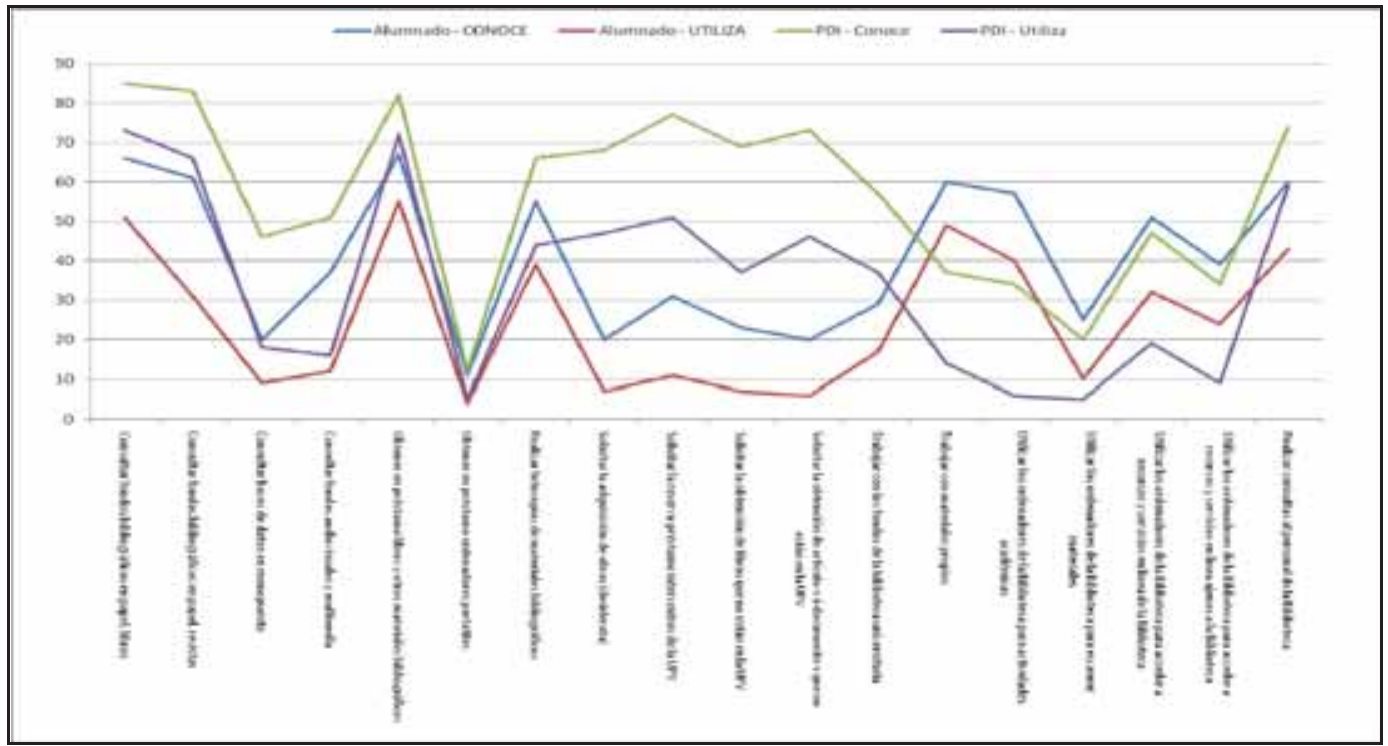

Gráfico 1: Alumnado y PDI. Conocimiento y uso de servicios presenciales.

En el caso del alumnado únicamente tres servicios presenciales son utilizados por, al menos, el 49\%. Estos son: consultar los fondos bibliográficos en papel, libros (51\%), obtener en préstamo libros y otros materiales bibliográficos $(55 \%)$ y trabajar con materiales propios (49\%). Por parte del PDI, son cinco los servicios presenciales que son utilizados por ese porcentaje: consultar los fondos bibliográficos en papel, libros (73\%), consultar los fondos bibliográficos en papel, revistas (66\%), obtener en préstamo libros y otros materiales bibliográficos $(72 \%)$ solicitar la reserva-préstamo intercentros de la UPV/EHU (51\%) y realizar consultas al personal de la Biblioteca $(59 \%)$.

fondos audiovisuales y multimedia; Obtener en préstamo libros y otros materiales bibliográficos; Obtener en préstamo ordenadores portátiles; Realizar fotocopias de materiales bibliográficos; Solicitar la adquisición de obras (desiderata); Solicitar la reserva-préstamo intercentros de la UPV; Solicitar la obtención de libros que no están en la UPV; Solicitar la obtención de artículo-s ó documento-s que no están en la UPV; Trabajar con los fondos de la biblioteca universitaria; Trabajar con materiales propios; Utilizar los ordenadores de la Biblioteca para actividades académicas; Utilizar los ordenadores de la Biblioteca para escanear materiales; Utilizar los ordenadores de la Biblioteca para acceder a recursos y servicios en línea de la Biblioteca; Utilizar los ordenadores de la Biblioteca para acceder a recursos y servicios en línea ajenos a la Biblioteca; Realizar consultas al personal de la Biblioteca 
La mayor diferencia entre los dos estamentos se muestra en los servicios que facilitan el acceso a los fondos de otros centros, bien sea dentro de la propia UPV/EHU bien externos a ésta. La menor diferencia se produce en el préstamo de ordenadores personales.

Una distribución muy parecida, pero con matices que llegan a marcar tendencias, se observa en el análisis por áreas de conocimiento, tal y como se puede observar a continuación.

En Ciencias Sociales y Jurídicas el alumnado ofrece resultados con variaciones pequeñas respecto a los resultados globales. Aún así, destaca el descenso de la consulta de libros y las fotocopias de los materiales bibliográficos.

Entre el PDI destacan los incrementos en el conocimiento y uso de la petición de adquisición de obras y en las diferentes opciones para la obtención de materiales fuera de su centro así como en trabajar con materiales propios.

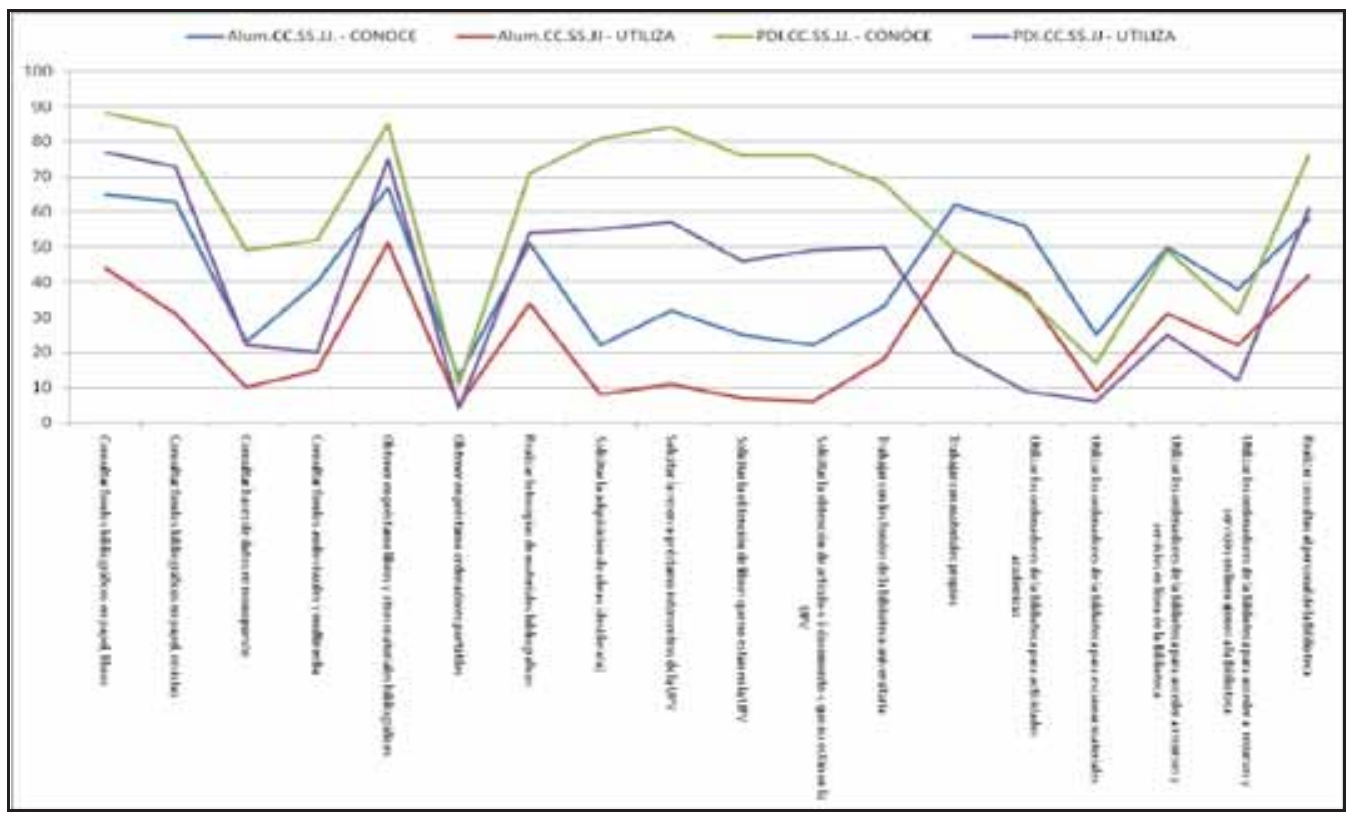

Gráfico 2: Ciencias Sociales y Jurídicas. Conocimiento y uso de servicios presenciales

En Ciencias de la Salud destaca, entre el alumnado, la escasa diferencia entre el conocimiento y el uso de la consulta de libros y el préstamo de libros y otros materiales. Destacan igualmente los incrementos en el trabajo con materiales propios y el uso de los ordenadores de la Biblioteca para actividades académicas y para acceder a recursos y servicios en línea ajenos a la Biblioteca. 


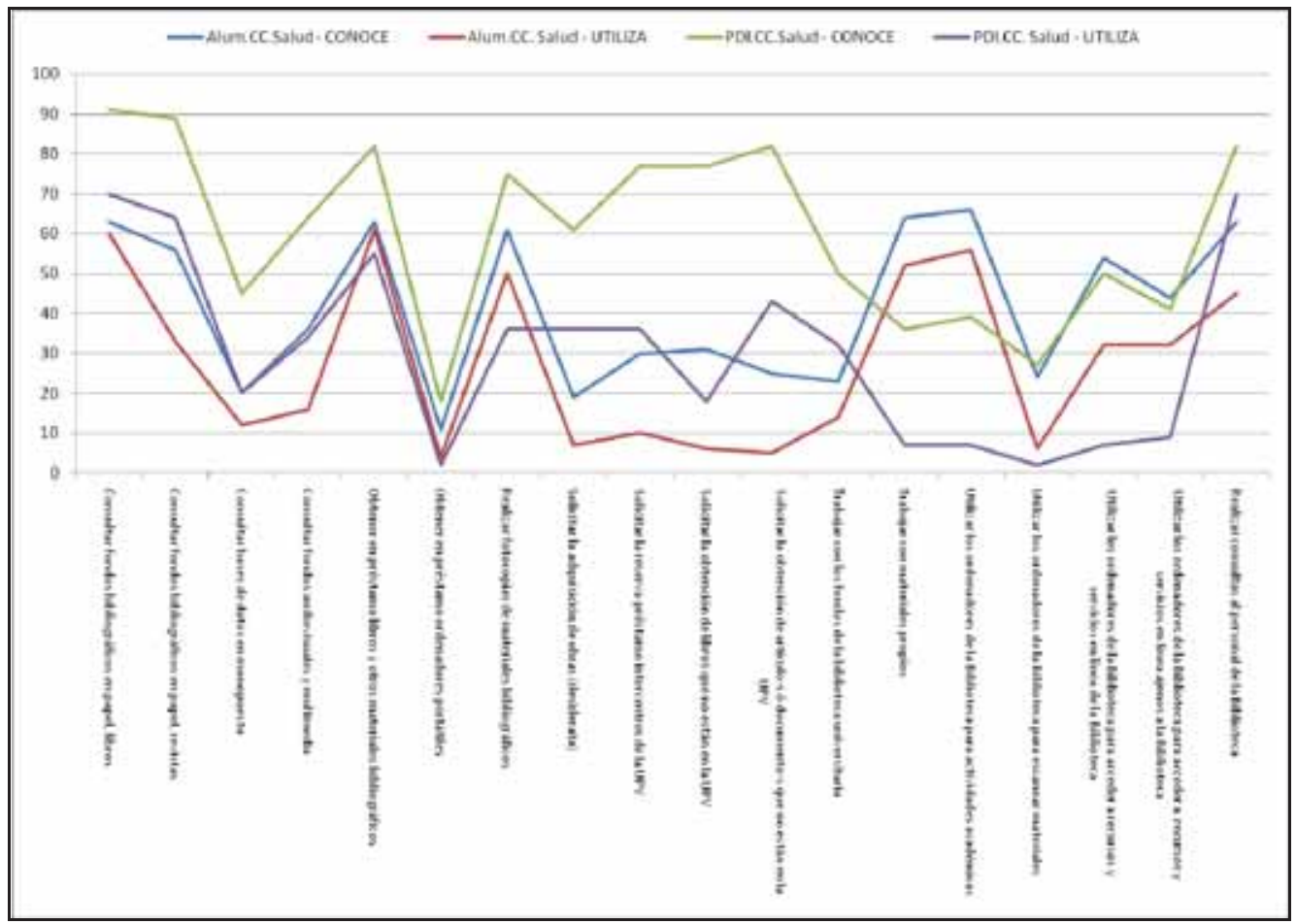

Gráfico 3: Ciencias de la Salud. Conocimiento y uso de servicios presenciales

Entre el PDI, destaca la disminución en los servicios de préstamo de fondos bibliográficos de la propia Biblioteca y la obtención de fondos de otros centros, bien de la propia UPV/EHU o bien externos y el uso de los ordenadores para acceder a recursos y servicios en línea ajenos a la Biblioteca. En sentido contrario, destacan los incrementos en los fondos audiovisuales y multimedia.

En Ciencias Experimentales se observa un incremento, en general, tanto del conocimiento como del uso de los servicios en ambos estamentos, aunque entre el alumnado es superior. 


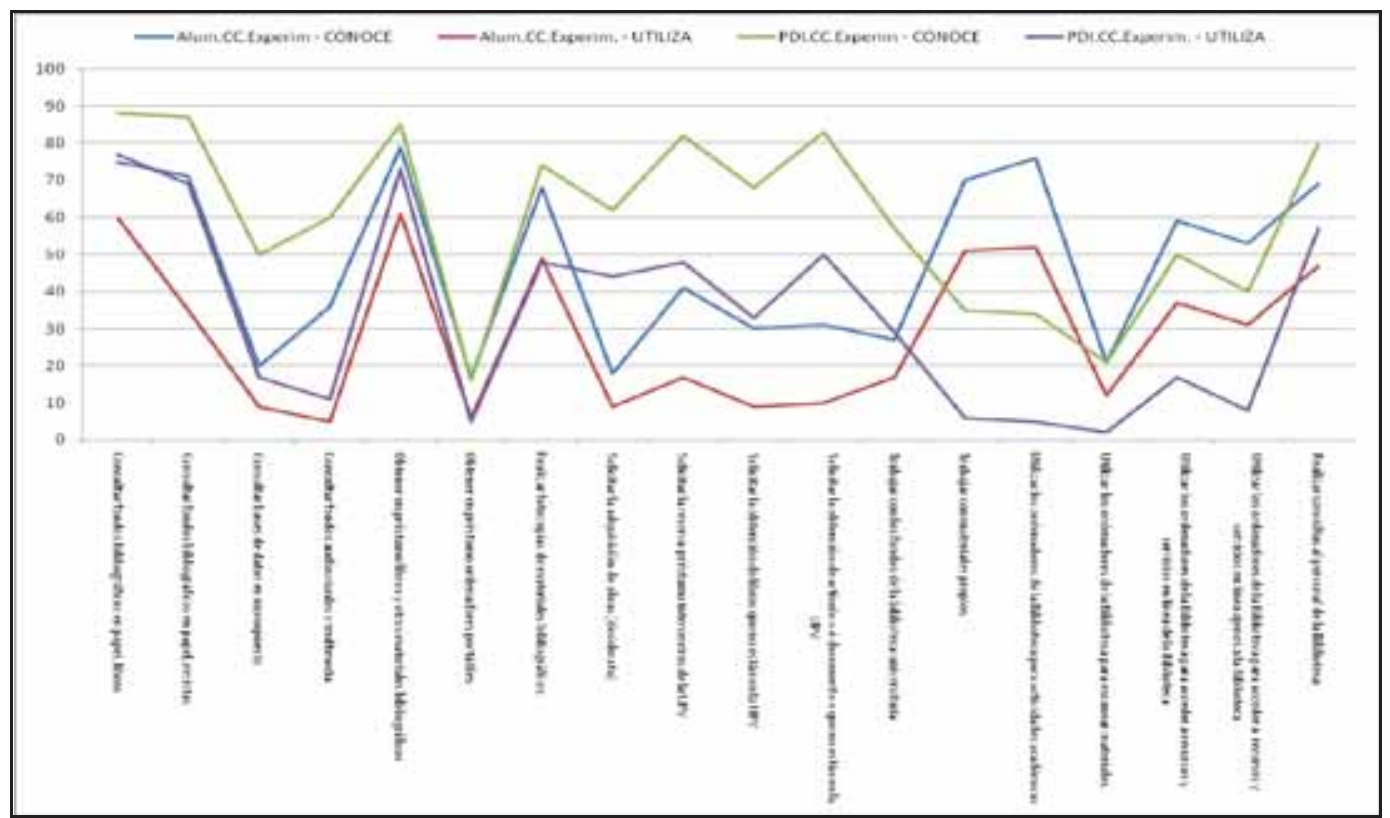

Gráfico 4: Ciencias Experimentales. Conocimiento y uso de servicios presenciales

En el alumnado destacan la consulta de los fondos (libros y revistas), el préstamo en el centro e intercentros y los diferentes usos de los ordenadores de la Biblioteca.

En el PDI, sin ser tan marcados, destacan los incrementos de la consulta de los fondos (libros y revistas) y el préstamo intercentros. Se incrementa la petición de obtención de artículos y documentos que no están en la UPV pero disminuye en el caso de libros. También disminuye el trabajo con materiales propios.

En el área de Humanidades se muestra una disminución de las distancias entre el conocimiento y el uso de algunos de los servicios. 


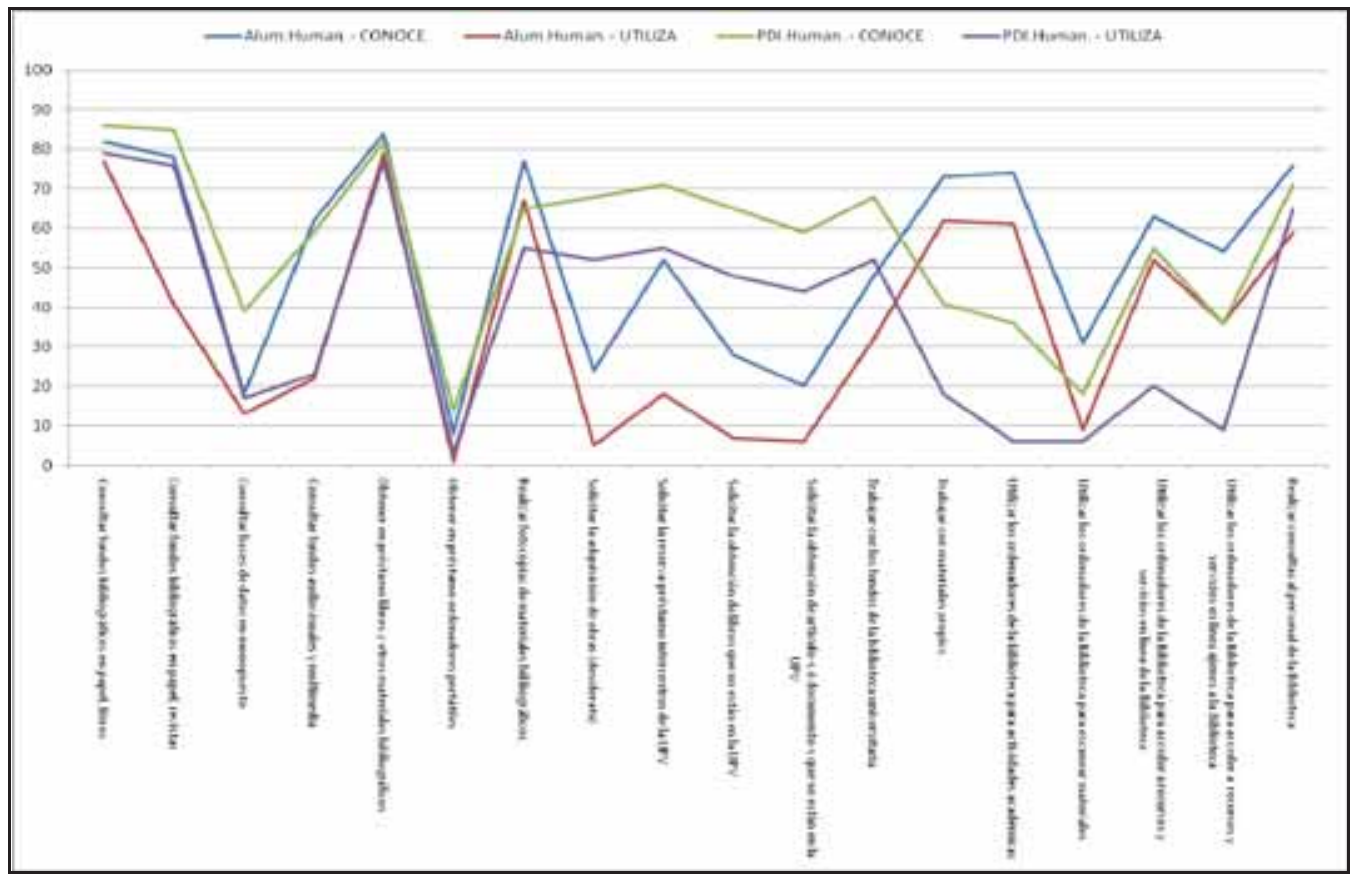

Gráfico 5: Humanidades. Conocimiento y uso de servicios presenciales

Es el caso del préstamo de fondos bibliográficos de la propia Biblioteca (tanto en el PDI como en el alumnado) y la obtención de fondos de otros centros, bien de la propia UPV/EHU (con fuerte incremento en su conocimiento por el alumnado) o bien externos. En ambos estamentos se recogen incrementos relacionados con los fondos audiovisuales y multimedia y con el trabajo con los fondos de la Biblioteca y, en menor medida, con los propios. El alumnado incrementa los porcentajes del uso de los ordenadores de la Biblioteca para actividades académicas.

El área de Enseñanzas Técnicas está marcada por un claro descenso. 


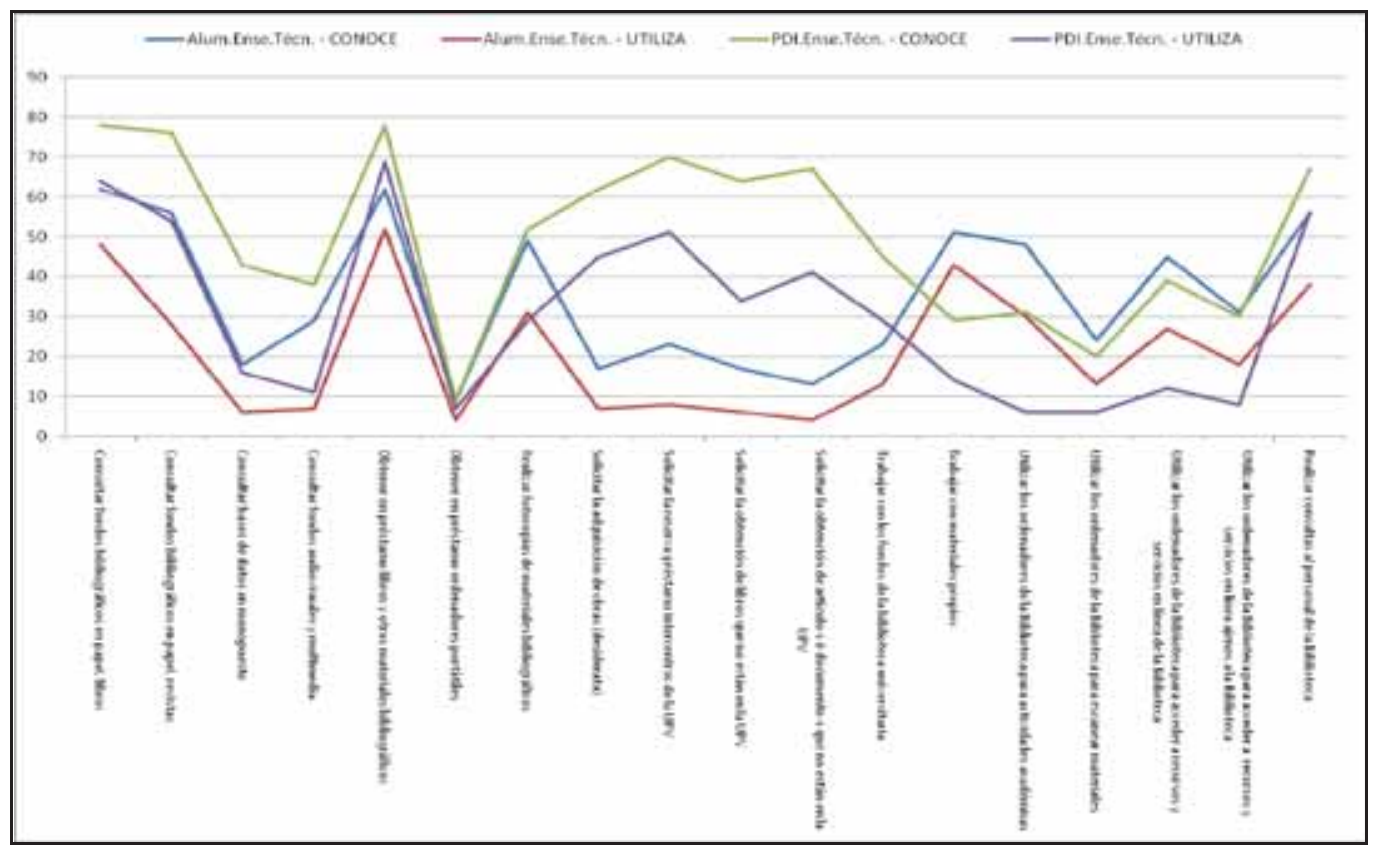

Gráfico 6: Enseñanzas Técnicas. Conocimiento y uso de servicios presenciales

Muestra una disminución generalizada de todos los porcentajes entre el alumnado salvo en el caso de la obtención de libros y otros materiales que no están en la UPV y en el escaneo de materiales. Entre el PDI se repite la situación con las salvedades del uso del préstamo de ordenadores portátiles y el uso de los ordenadores de la Biblioteca para el escaneo de materiales.

Por otra parte, el análisis de los resultados globales por áreas muestra cómo en Humanidades hay, en general y con alguna salvedad, un mayor conocimiento de estos servicios. En el extremo contrario, el área que presenta el grado de conocimiento más bajo es Enseñanzas Técnicas. 


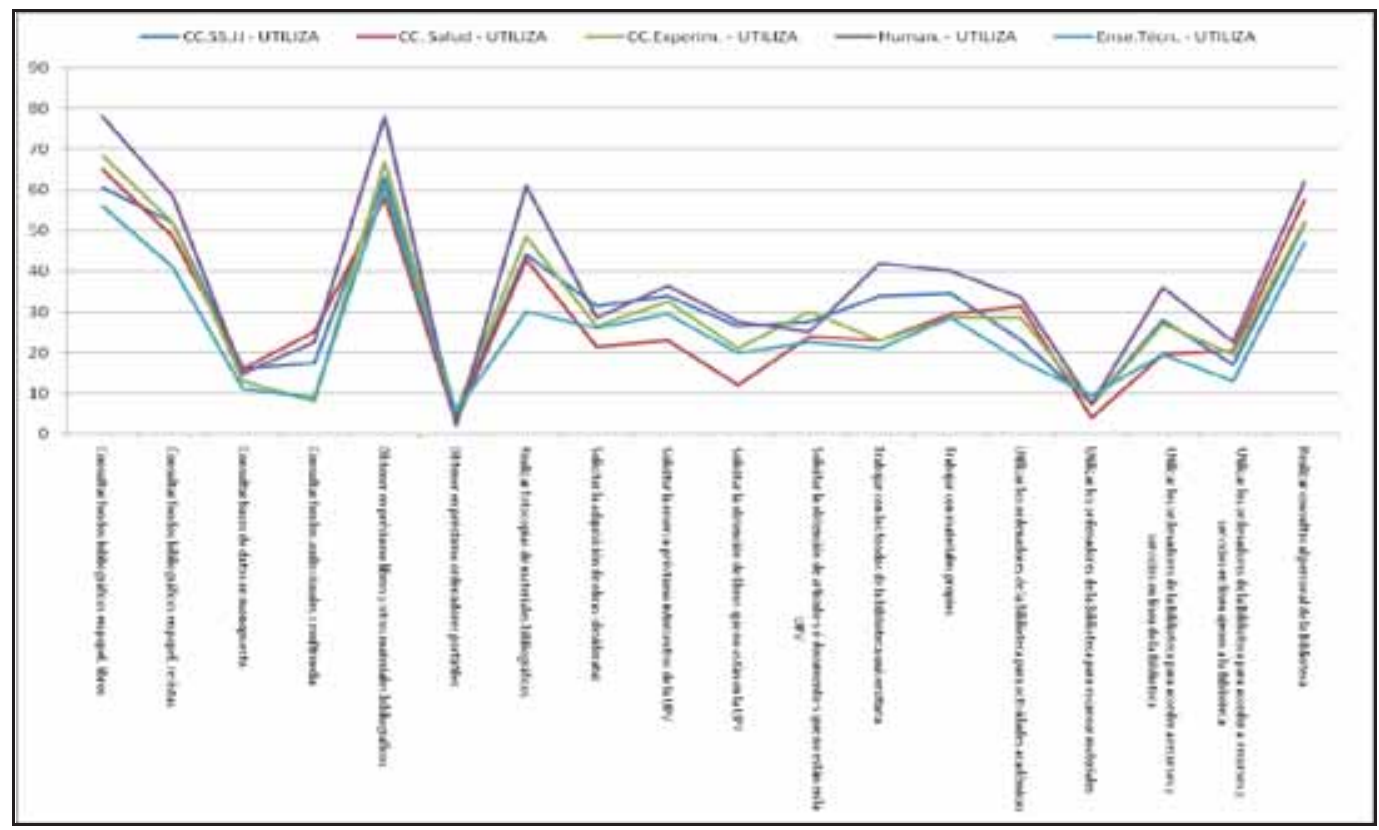

Gráfico 7: Conocimiento de servicios presenciales por áreas.

En cuanto al uso que se hace de estos servicios presenciales, vuelve a ser el área de Humanidades el que alcanza los porcentajes más elevados, en general y con alguna salvedad (p.e. solicitar la obtención de artículo-s o documento-s que no están en la UPV/EHU). Enseñanzas Técnicas y Ciencias de la Salud son las áreas que muestran los porcentajes más bajos con mayor frecuencia. 


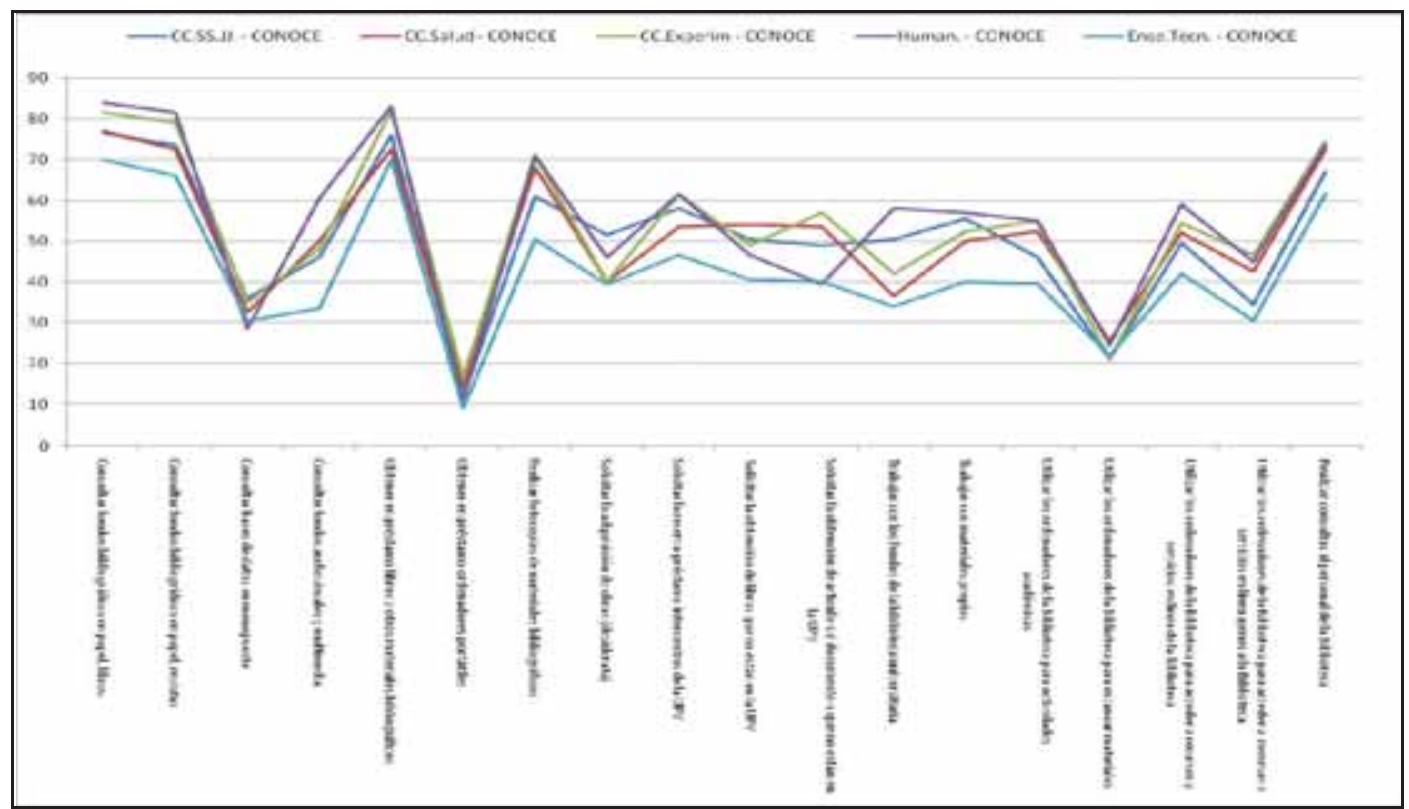

Gráfico 8: Utilización de servicios presenciales por áreas.

Al realizar el análisis del grado de conocimiento del alumnado por áreas se observa cómo Humanidades y Ciencias Experimentales muestran un mayor conocimiento general de estos servicios presenciales. El área de Enseñanzas Técnicas, por el contrario, es el que muestra los niveles más bajos. 


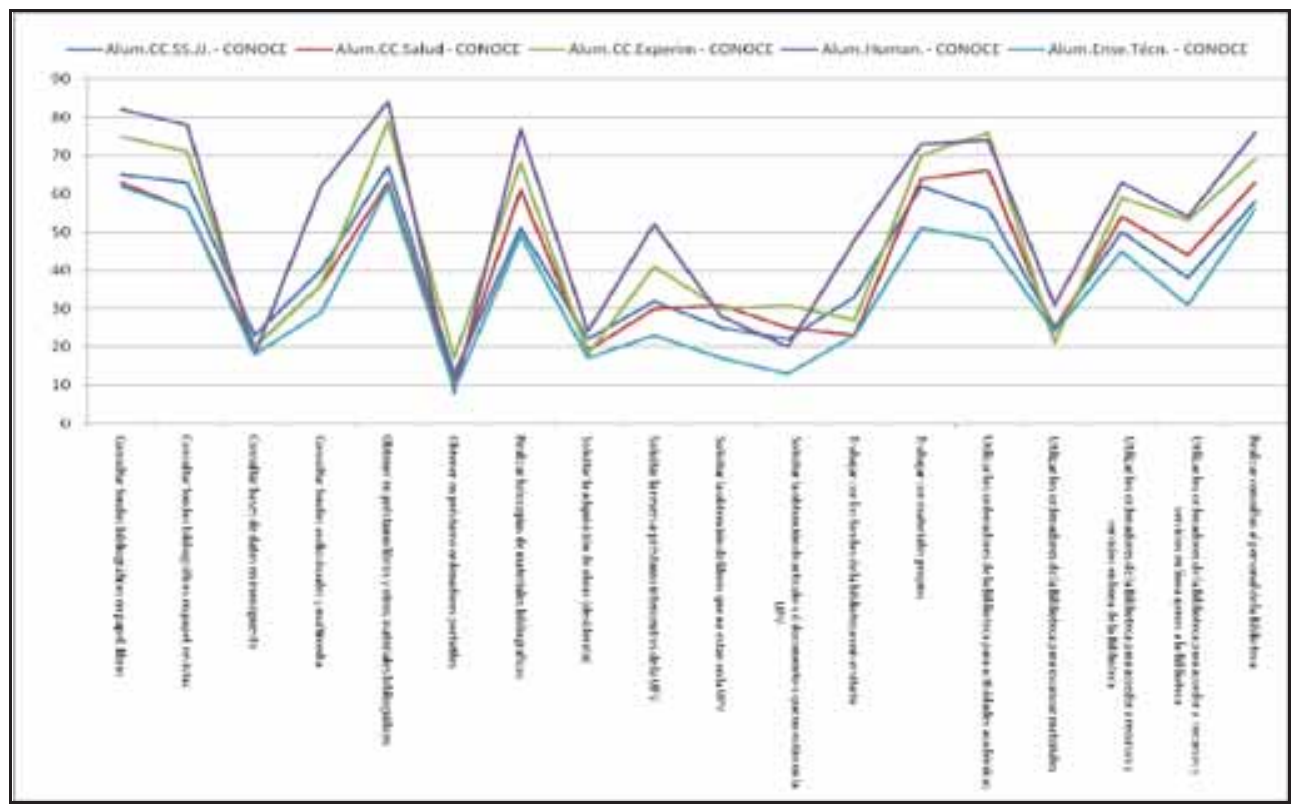

Gráfico 9: Alumnado. Conocimiento de servicios presenciales por áreas.

Las diferencias en el porcentaje de conocimiento por áreas se reducen especialmente en la consulta de bases de datos en monopuesto, en obtener ordenadores portátiles en préstamo, en solicitar la adquisición de obras y en utilizar los ordenadores de la Biblioteca para escanear materiales. La mayor diferencia, por el contrario, se da en utilizar los ordenadores de la Biblioteca para actividades académicas y en fotocopiar materiales bibliográficos.

En cuanto a la utilización de estos servicios por el alumnado: 


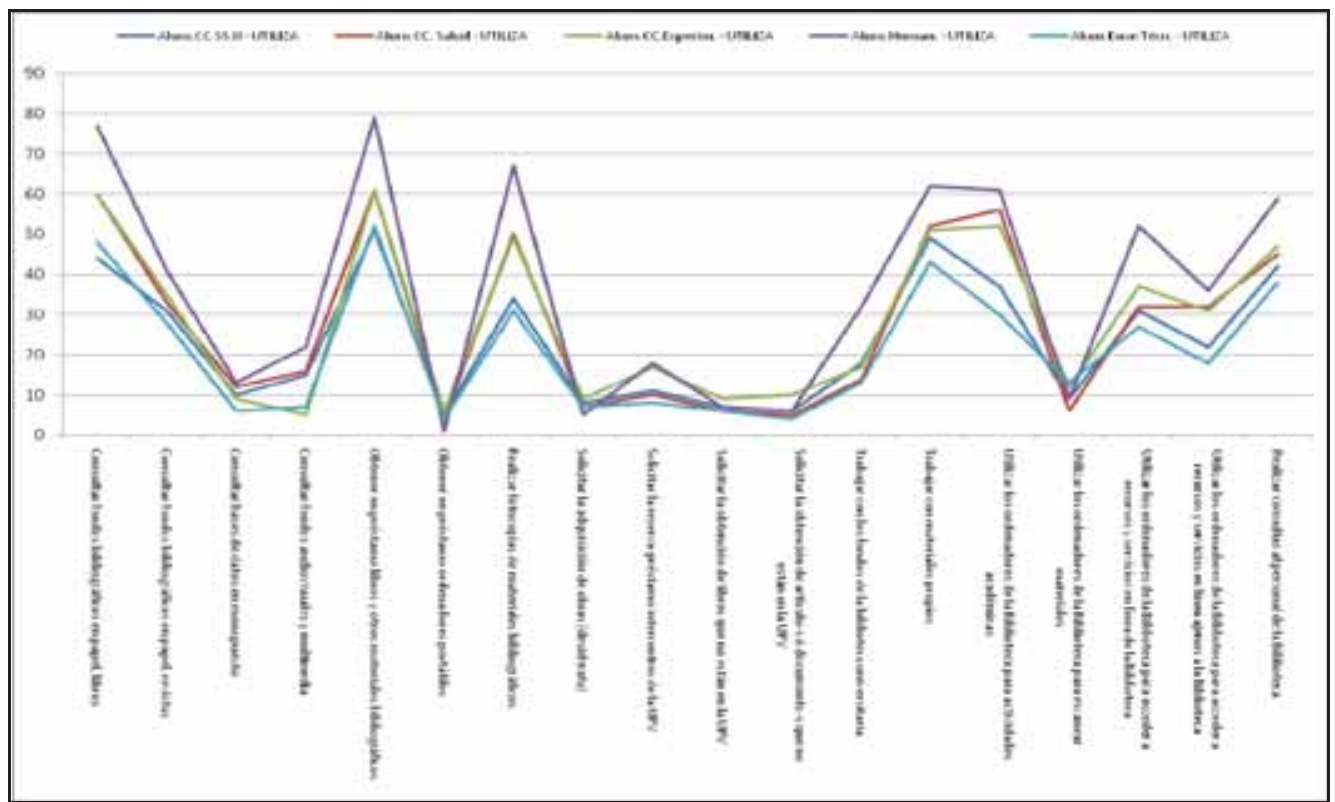

Gráfico 10: Alumnado. Utilización de servicios presenciales por áreas.

También son las áreas de Humanidades y Ciencias Experimentales las que muestran, en general los porcentajes más altos, con la inclusión del área de Ciencias de la Salud en algunos de ellos. En estas tres áreas es especialmente reseñable (49\% o más) el incremento en el uso de los servicios de realizar fotocopias de materiales bibliográficos, trabajar con materiales propios y utilizar los ordenadores de la Biblioteca para actividades académicas. El área de Humanidades, además, incrementa el uso de los ordenadores de la biblioteca para acceder a los recursos y servicios en línea de la Biblioteca y el hacer consultas al personal de la Biblioteca.

El área de Enseñanzas Técnicas es, habitualmente, el que menor uso hace de ellos salvo el escaneo de materiales en el que es el primero.

Por su parte, entre el PDI el área de Enseñanzas Técnicas es, en general, el que muestra un menor conocimiento de estos servicios. El resto de las áreas mantienen habitualmente unos niveles de conocimiento superiores a los de aquél, intercambiándose las posiciones entre ellos. 


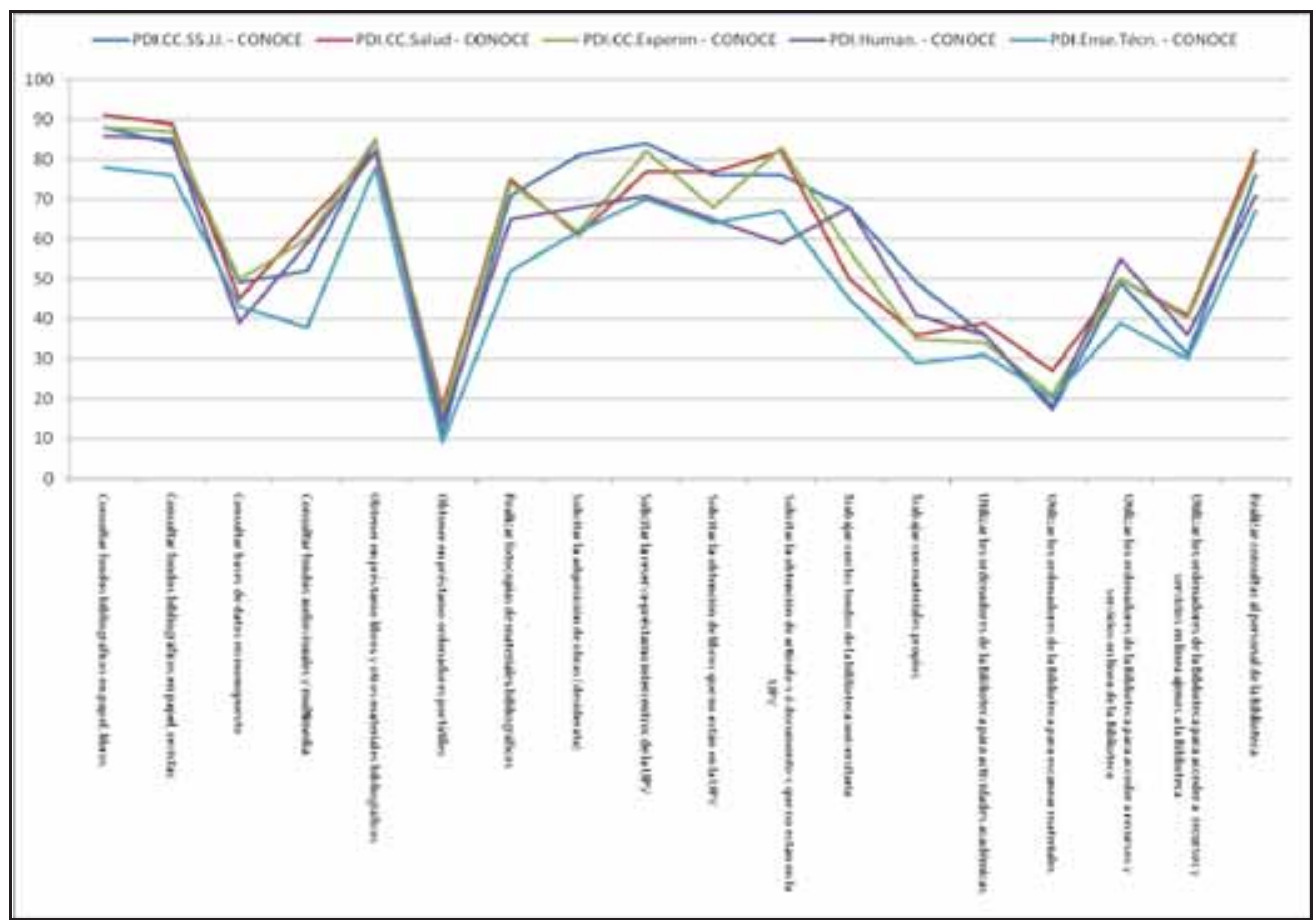

Gráfico 11: PDI. Conocimiento de servicios presenciales por áreas.

En cuanto al uso de estos servicios por parte del PDI, las áreas de Ciencias Sociales y Jurídicas y de Humanidades son, en general, las que alcanzan un mayor grado de uso. Logran superar el $49 \%$ en el uso de servicios como: realizar fotocopias de materiales bibliográficos, solicitar la adquisición de obras (desideratas), solicitar la reservapréstamo intercentros UPV y trabajar con los fondos de la Biblioteca. 


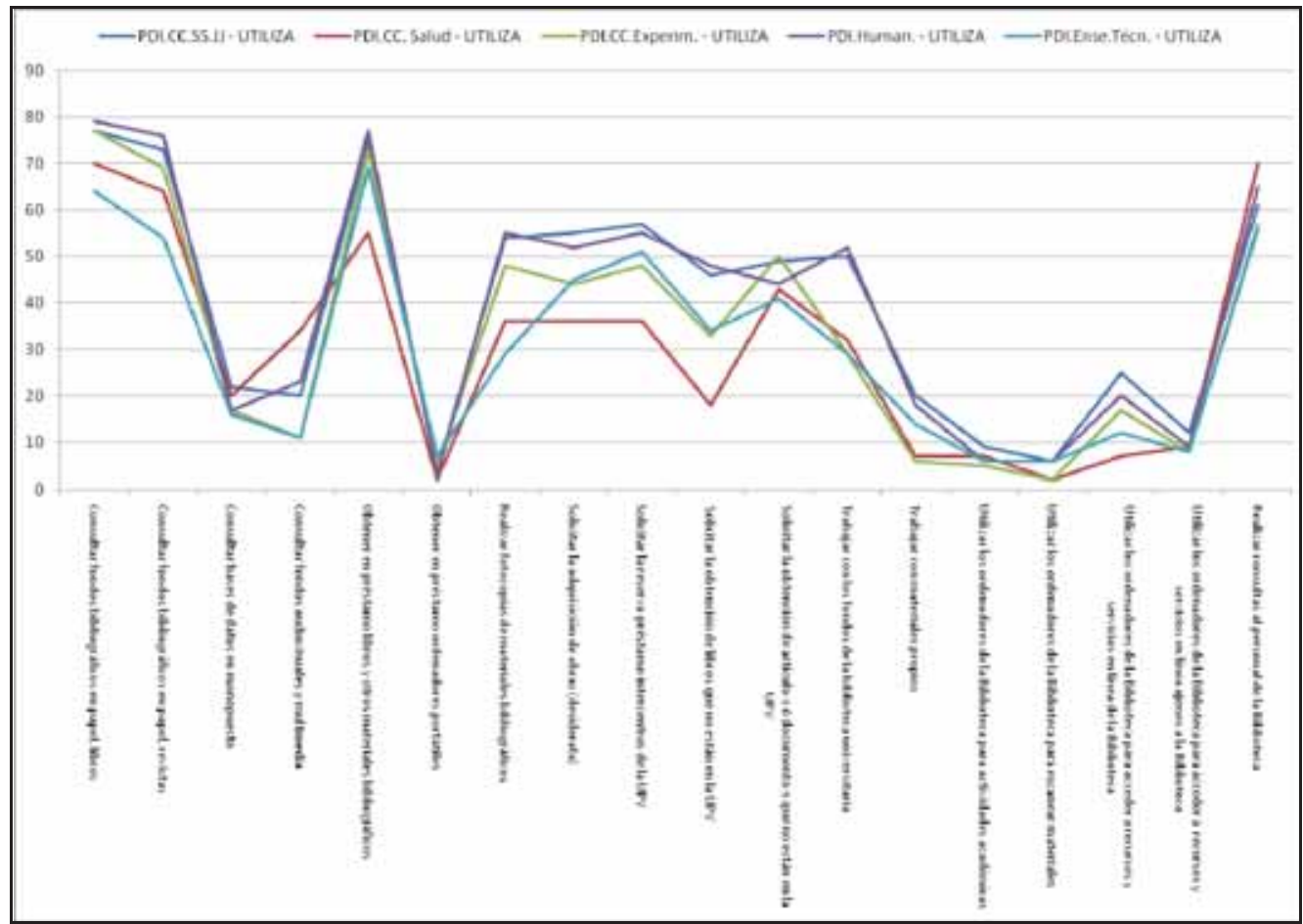

Gráfico 12: PDI. Utilización de servicios presenciales por áreas.

Por su parte, el área de Ciencias de la Salud muestra, en general, el uso más bajo con algunas salvedades. Le siguen Ciencias Experimentales y Enseñanzas Técnicas que se intercambian su posición con frecuencia. Aunque también logran superar el 49\%: Enseñanzas Técnicas en solicitar la reserva-préstamo intercentros UPV y Ciencias Experimentales en solicitar la obtención de articulo-s o documentos-s que no están en la UPV.

\section{CONOCIMIENTO Y USO DE SERVICIOS NO PRESENCIALES}

El análisis de los servicios no presenciales ${ }^{4}$ nos muestra cómo el conocimiento y uso de éstos es claramente muy superior entre el PDI. A pesar de eso, tan solo se muestran

${ }^{4}$ Los servicios no presenciales identificados fueron: Consultar y-o actualizar el propio registro de usuario; Consultar el catálogo en línea; Solicitar la adquisición de obras (desiderata); Solicitar la reservapréstamo intercentros de la UPV; Solicitar la obtención de libros que no están en la UPV; Solicitar la obtención de artículos ó documentos que no están en la UPV; Consultar las nuevas adquisiciones en el catálogo de la Biblioteca; Consultar-utilizar las guías de la Biblioteca; Consultar los fondos bibliográficos en línea, libros electrónicos; Consultar los fondos bibliográficos en línea, revistas 
porcentajes de uso superiores al 50\% en tres de estos servicios: consultar el catálogo en línea $(69 \%)$, solicitar la reserva-préstamo intercentros de la UPV/EHU (51\%) y consultar revistas electrónicas $(53 \%)$.

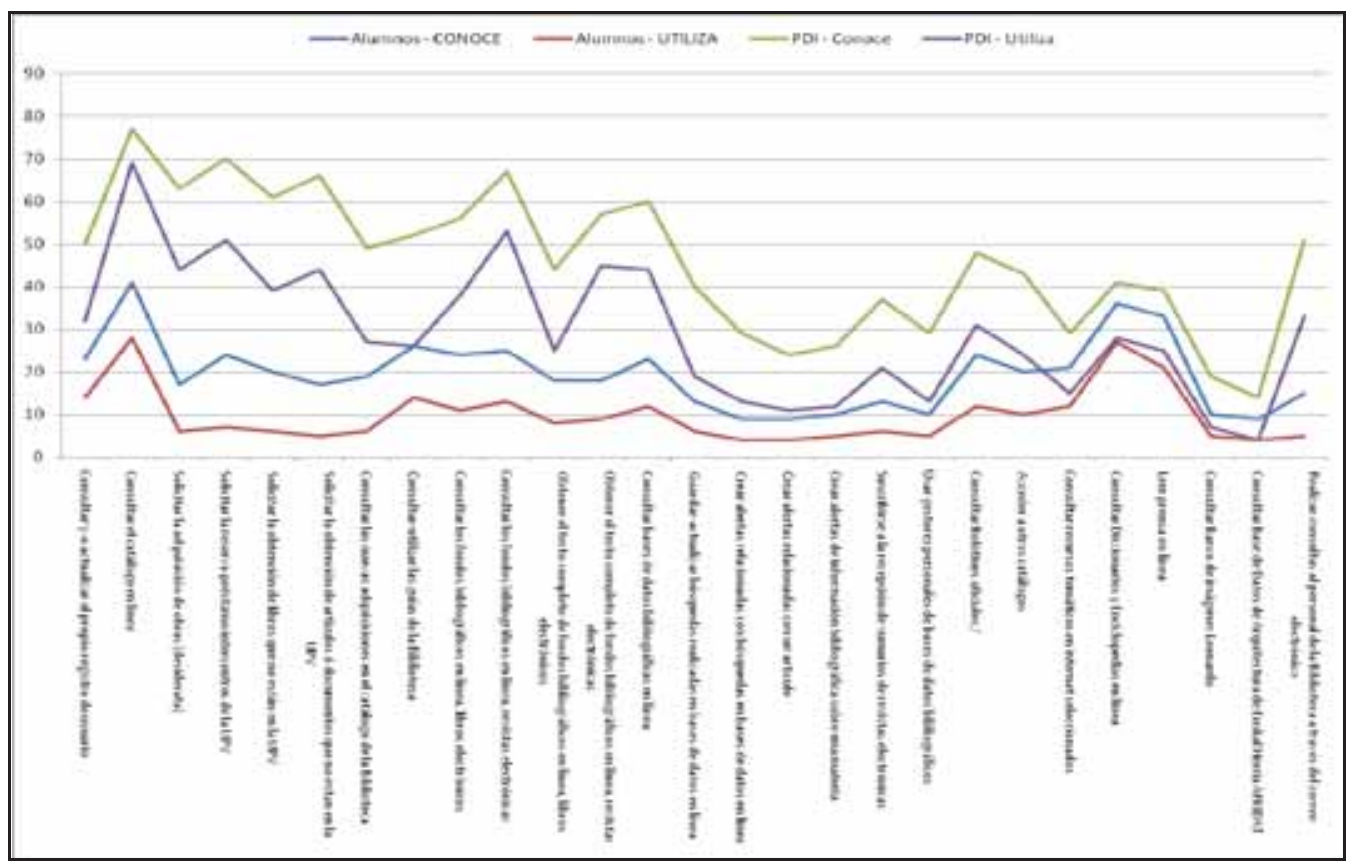

Gráfico 13: Alumnado y PDI. Conocimiento y uso de servicios no presenciales.

En el caso del alumnado, los porcentajes de uso son realmente bajos. Tan solo en dos casos se llega a superar el $25 \%$ : consultar el catálogo en línea $(28 \%)$ y consultar diccionarios y enciclopedias en línea (27\%). En la mayoría de estos servicios los porcentajes de uso se mantienen por debajo del $10 \%$ o lo superan mínimamente.

electrónicas; Obtener el texto completo de fondos bibliográficos en línea, libros electrónicos; Obtener el texto completo de fondos bibliográficos en línea, revistas electrónicas; Consultar bases de datos bibliográficas en línea; Guardar-actualizar búsquedas realizadas en bases de datos en línea; Crear alertas relacionadas con búsquedas en bases de datos en línea; Crear alertas relacionadas con un artículo; Crear alertas de información bibliográfica sobre una materia; Suscribirse a la recepción de sumarios de revistas electrónicas; Usar gestores personales de bases de datos bibliográficos; Consultar Boletines oficiales; Acceder a otros catálogos; Consultar recursos temáticos en Internet seleccionados; Consultar Diccionarios y Enciclopedias en línea; Leer prensa en línea; Consultar Banco de imágenes Leonardo; Consultar Base de Datos de Arquitectura de Euskal Herria ARKIDAT; Realizar consultas al personal de la Biblioteca a través del correo electrónico 
En los siguientes gráficos se puede comprobar las variaciones que se presentan en el conocimiento y uso de los servicios no presenciales de la Biblioteca entre las diferentes áreas.

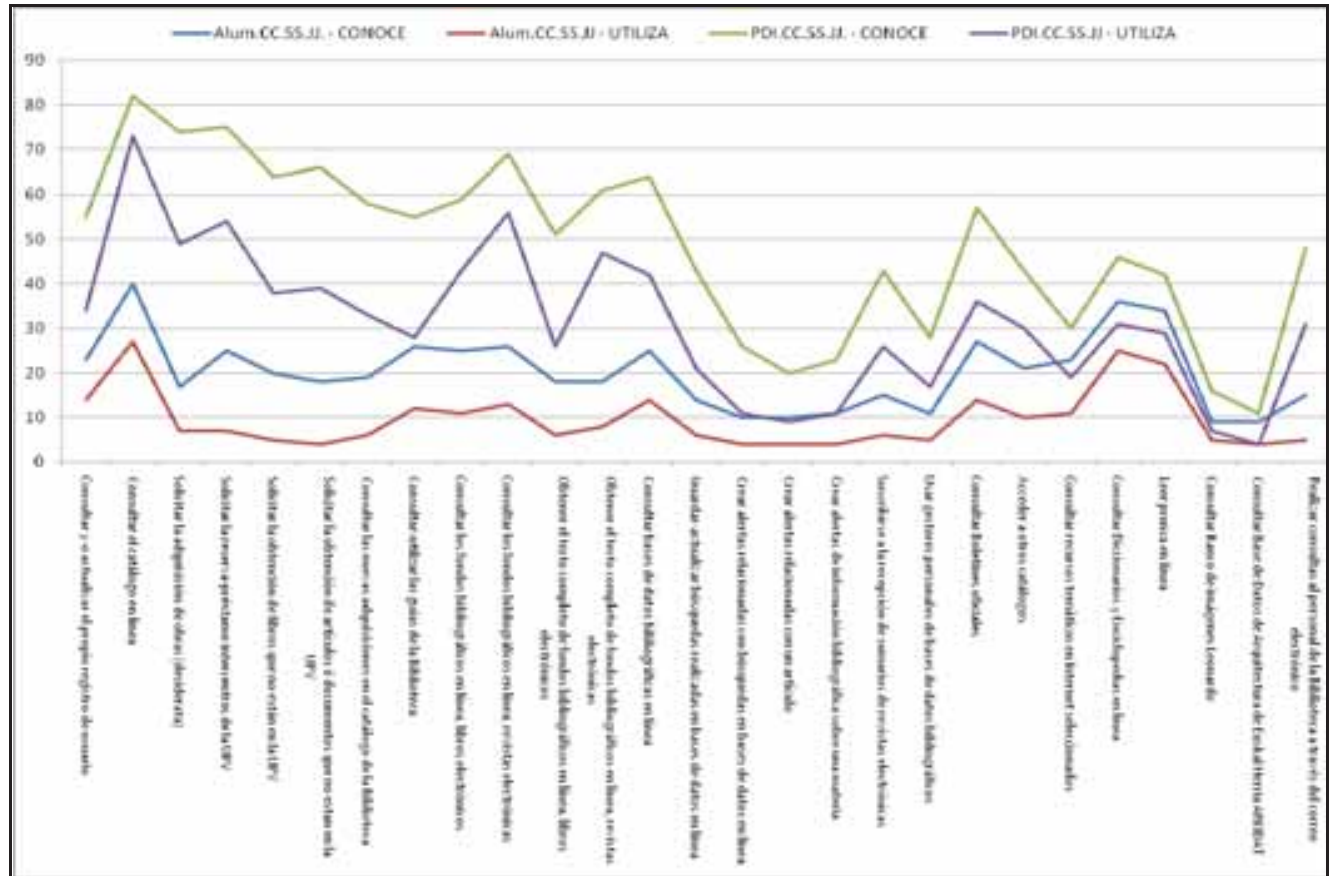

Gráfico 14: Ciencias Sociales y Jurídicas. Conocimiento y uso de servicios no presenciales

El área de Ciencias Sociales y Jurídicas muestra variaciones pequeñas respecto a los resultados generales entre el alumnado. Entre el PDI se produce un incremento en general y con variaciones superiores a las del alumnado. Destacan los incrementos en la solicitud de adquisición de obras, en la consulta de las nuevas adquisiciones en el catálogo de la biblioteca, en la suscripción a los sumarios de revistas electrónicas y en la consulta de boletines oficiales. En sentido contrario, disminuyen los porcentajes relacionados con la creación de alertas. 


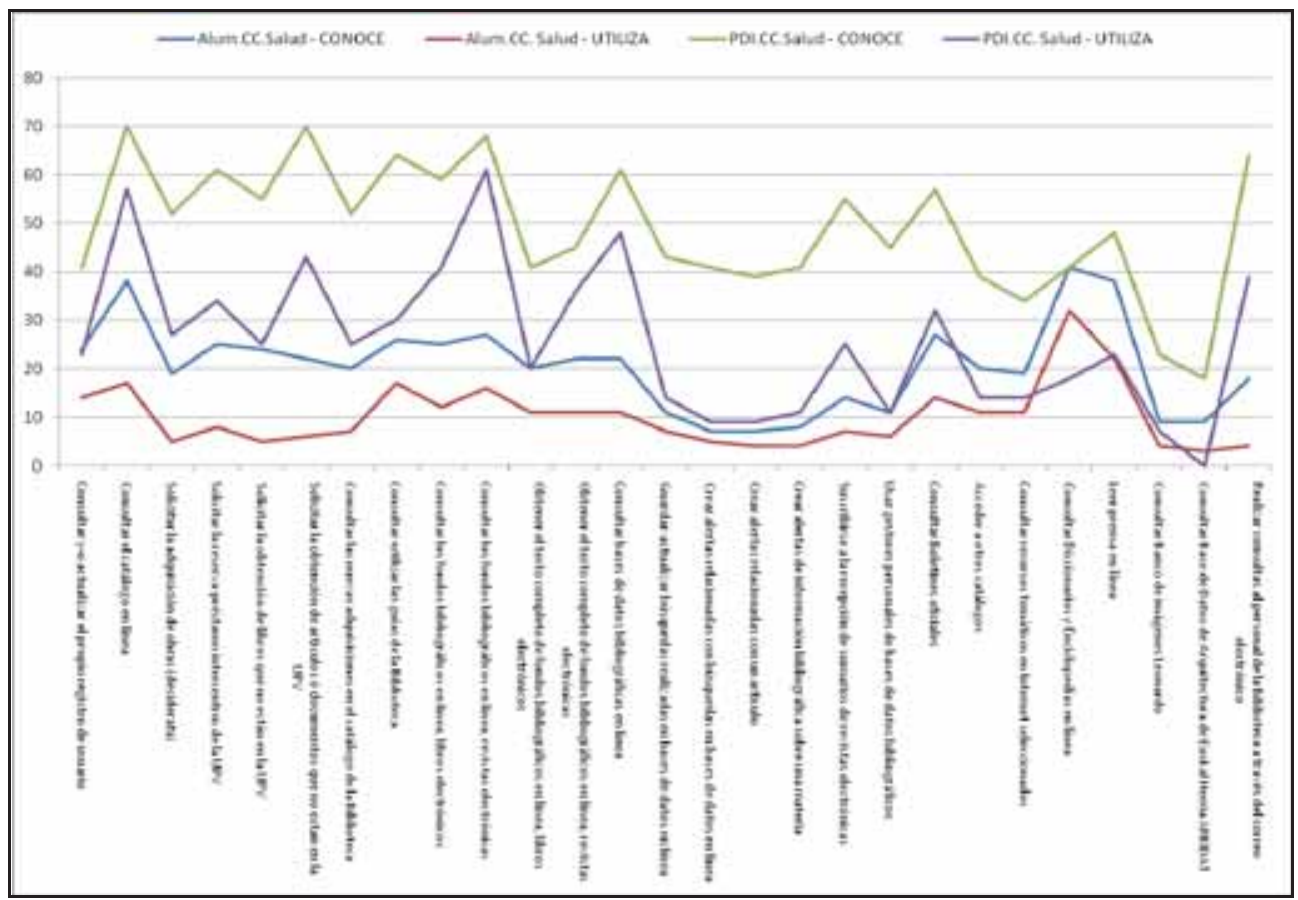

Gráfico 15: Ciencias de la Salud. Conocimiento y uso de servicios no presenciales

En el área de Ciencias de la Salud destacan dos resultados paradójicos relacionados con el uso de las revistas electrónicas en línea por parte del PDI. Por un lado incrementa su consulta pero, por otro, disminuye la obtención del texto completo de las mismas. En el alumnado destaca el incremento de la consulta de diccionarios y enciclopedias en línea y, en sentido contrario, la disminución de forma importante de la consulta del catálogo en línea. 


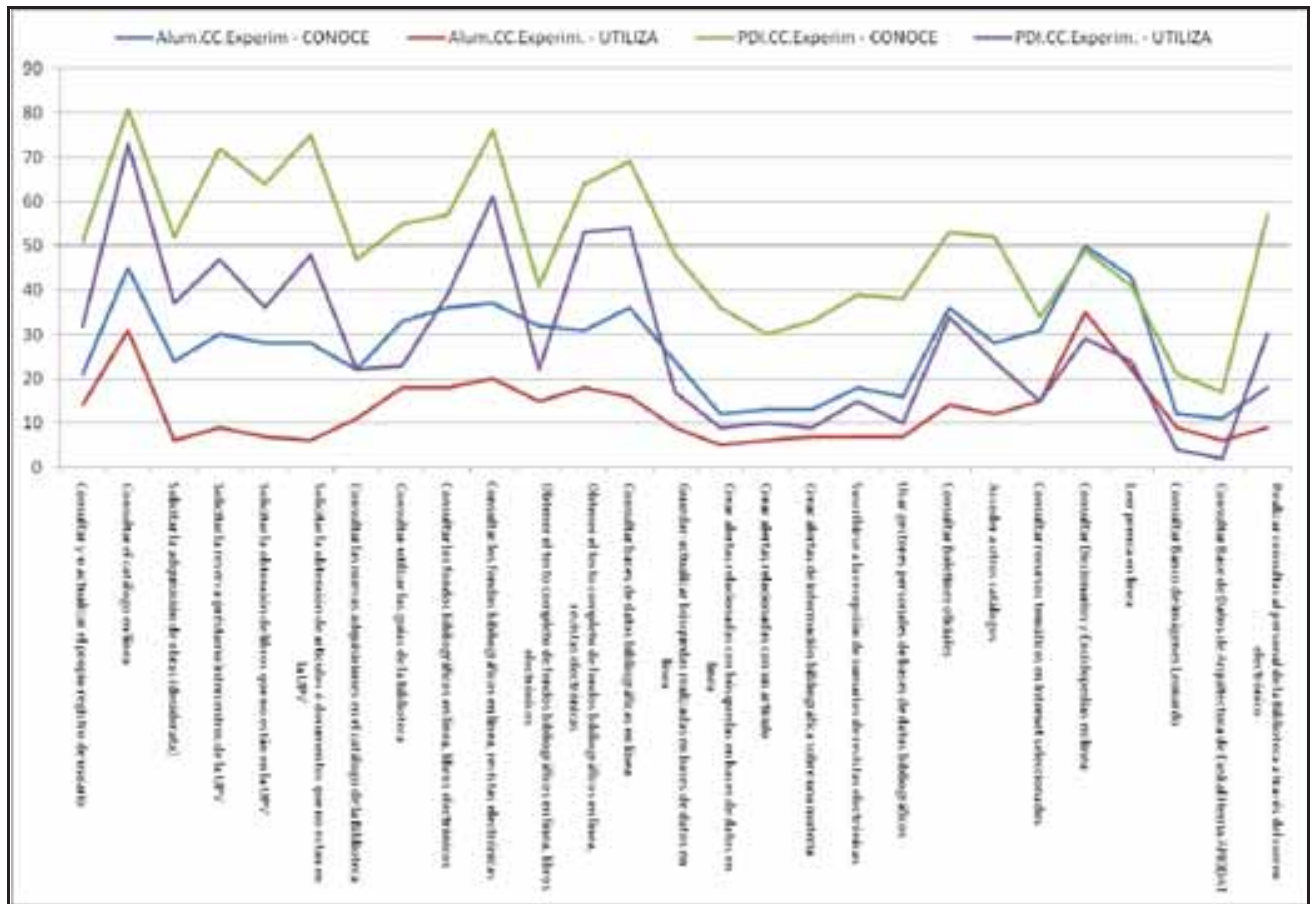

Gráfico 16: Ciencias Experimentales. Conocimiento y uso de servicios no presenciales

En Ciencias Experimentales destaca el incremento en el PDI de la consulta de revistas electrónicas en línea, así como de la obtención del texto completo de éstas y la consulta de bases de datos en línea. En el caso del alumnado se percibe un incremento del uso de los recursos en línea: consulta de libros y revistas electrónicas, obtención del texto completo tanto de unos como de otras, consultar bases de datos bibliográficos en línea y consultar diccionarios y enciclopedias en línea (superando al PDI en este último). 


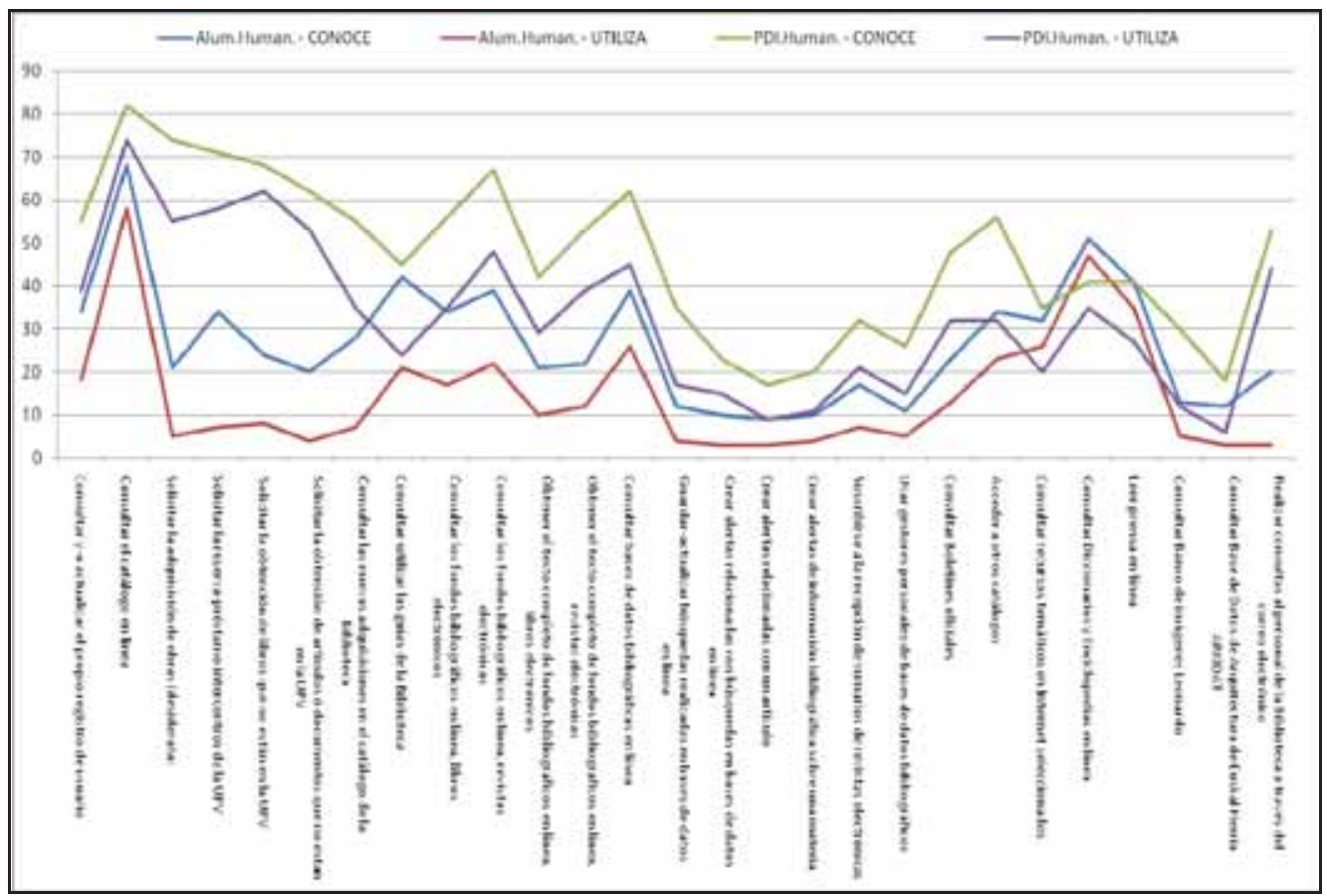

Gráfico 17: Humanidades. Conocimiento y uso de servicios no presenciales

En el área de Humanidades, entre el PDI se incrementa el uso de la petición de adquisición de obras (desiderata), la reserva-préstamo intercentros de la UPV/EHU, la solicitud de libros que no están en la UPV/EHU, el acceso a otros catálogos y la consulta de diccionarios y enciclopedias en línea. Disminuyen algunos de los servicios relacionados con recursos electrónicos en línea. Por parte del alumnado se incrementa el uso de: guías de la Biblioteca; consulta de fondos bibliográficos en línea, tanto libros como revistas, y obtención del texto en ambos casos; consulta de bases de datos bibliográficas en línea; acceso a otros catálogos; consulta de recursos temáticos de Internet seleccionados; consulta de diccionarios y enciclopedias y leer prensa en línea (superando en estas tres últimas al PDI). 


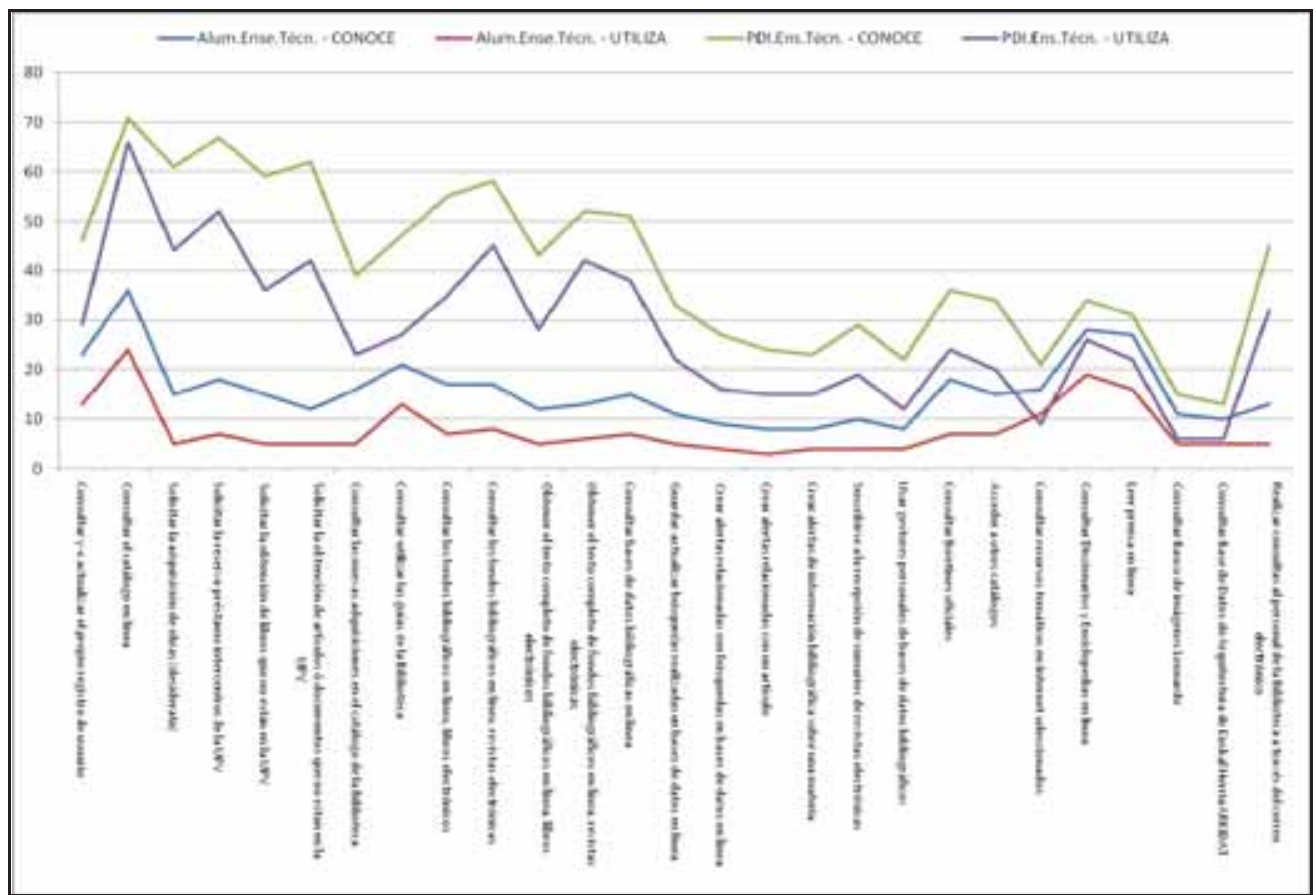

Gráfico 18: Enseñanzas Técnicas. Conocimiento y uso de servicios no presenciales

En las Enseñanzas Técnicas tanto el alumnado como el PDI muestran unos perfiles básicamente similares a los generales, pero con una disminución generalizada de los porcentajes. Entre el alumnado, estos descensos son más acusados en: consulta de fondos bibliográficos en línea, tanto libros como revistas electrónicas; consulta de bases de datos bibliográficas en línea; consultar boletines oficiales; consultar diccionarios y enciclopedias en línea; y leer prensa en línea. En el caso del PDI, los descensos más marcados, superiores incluso que los del alumnado se obtienen en la consulta de revistas electrónicas y en la consulta de bases de datos bibliográficas en línea. Muestra, por el contrario, incrementos en la obtención del texto completo de libros electrónicos, en guardar-actualizar búsquedas en bases de datos, y en la creación de los diferentes tipos de alertas. 


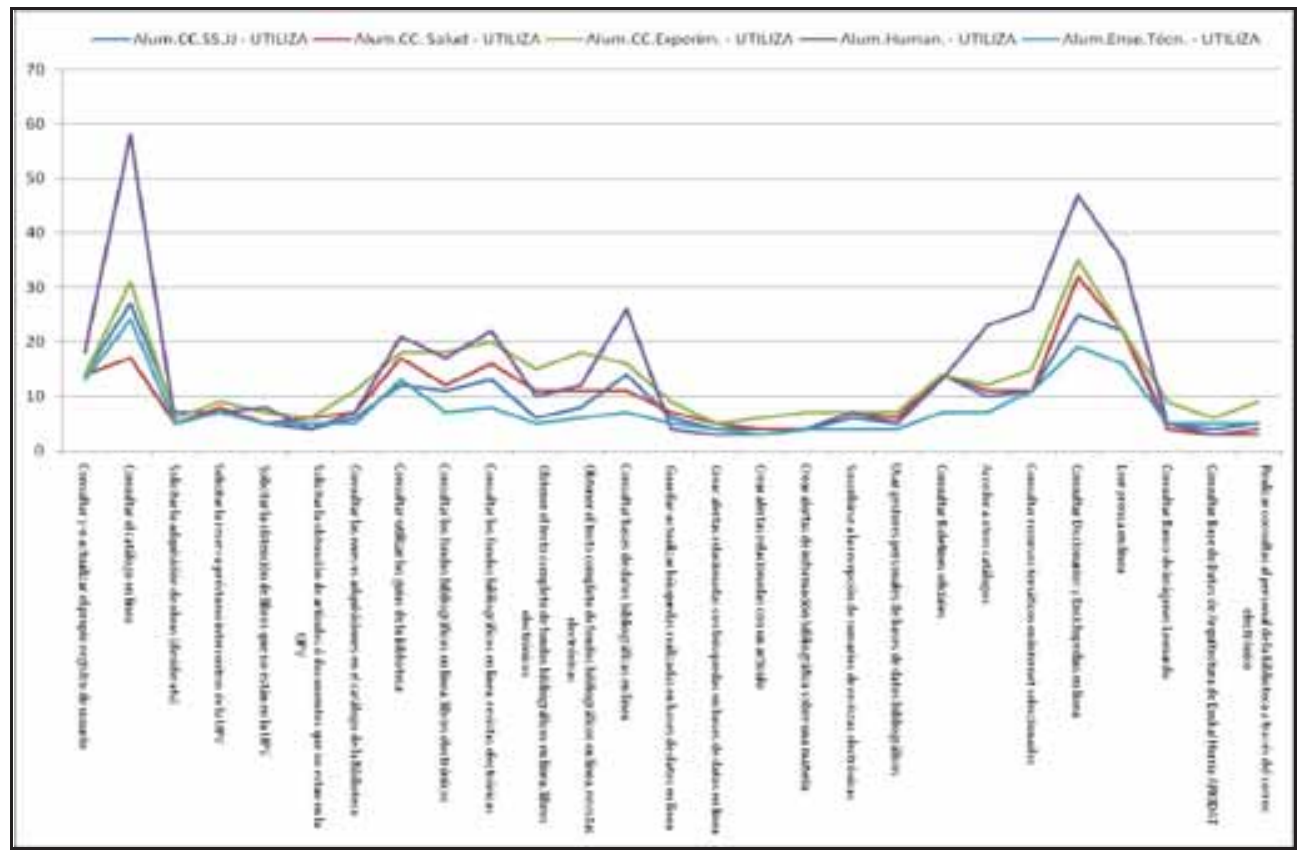

Gráfico 19: Alumnado. Conocimiento de servicios no presenciales por áreas.

Analizado el conocimiento de los servicios no presenciales de la Biblioteca entre el alumnado, las áreas de Humanidades y Ciencias Experimentales muestran, en general, mayores porcentajes. Enseñanzas Técnicas, por el contrario, es el área que muestra el menor grado de conocimiento en prácticamente la totalidad de los servicios.

Las menores variaciones entre las áreas se producen en la creación de los diferentes tipos de alertas y en la consulta de Leonardo y Arkidat. 


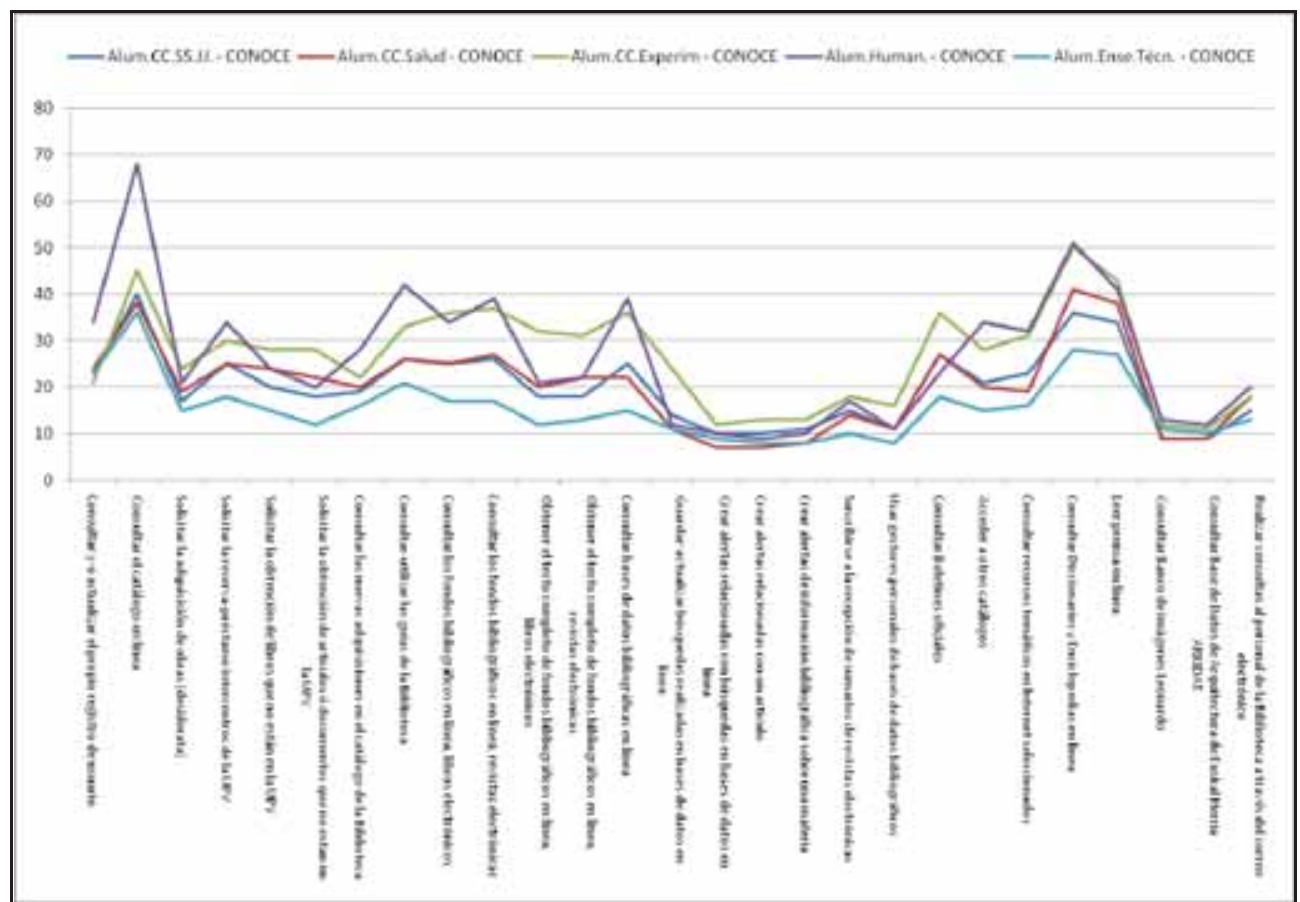

Gráfico 20: Alumnado. Utilización de servicios no presenciales por áreas.

En el caso del uso de estos servicios, las áreas de Humanidades y Ciencias Experimentales muestran, en general los mayores porcentajes. Enseñanzas Técnicas y, en menor medida, Ciencias Sociales y Jurídicas son las que menor uso hacen de los mismos. Las mayores variaciones se muestran en los incrementos por el área de Humanidades en: consultar el catálogo en línea, consultar bases de datos bibliográficas en línea, acceder a otros catálogos, consultar recursos temáticos en Internet seleccionados, consultar diccionarios y enciclopedias en línea y leer prensa en línea 


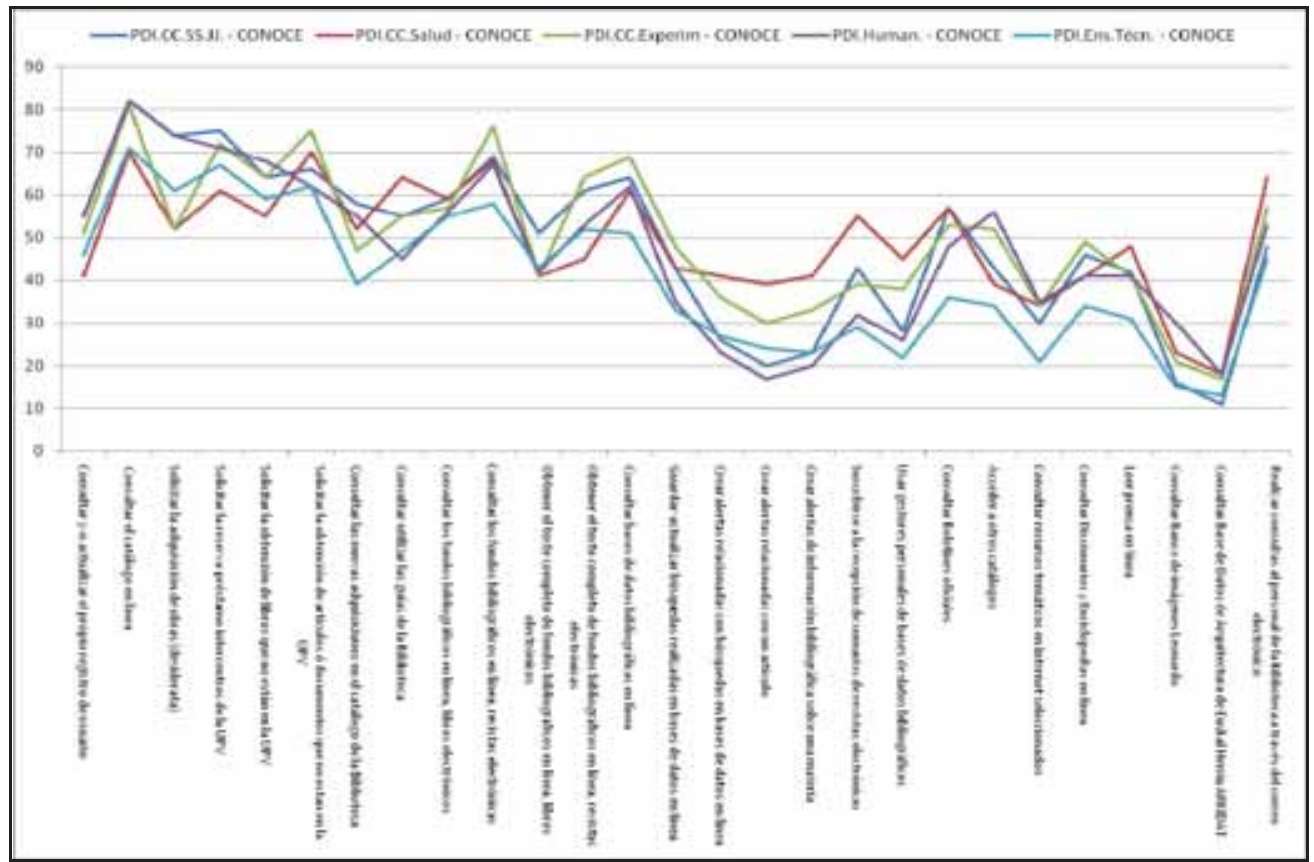

Gráfico 21: PDI. Conocimiento de servicios no presenciales por áreas.

En el análisis de los datos del PDI, el área de Enseñanzas Técnicas es el que muestra con más frecuencia el menor grado de conocimiento de los servicios no presenciales de la Biblioteca. Las áreas de Humanidades y Ciencias Sociales y Jurídicas muestran mayores variaciones entre los resultados de los servicios que más conocen y aquellos que menos. Por último y pese a las lógicas fluctuaciones, Ciencias Experimentales y Ciencias de la Salud, mantienen menores variaciones con niveles altos de conocimiento, en general. 


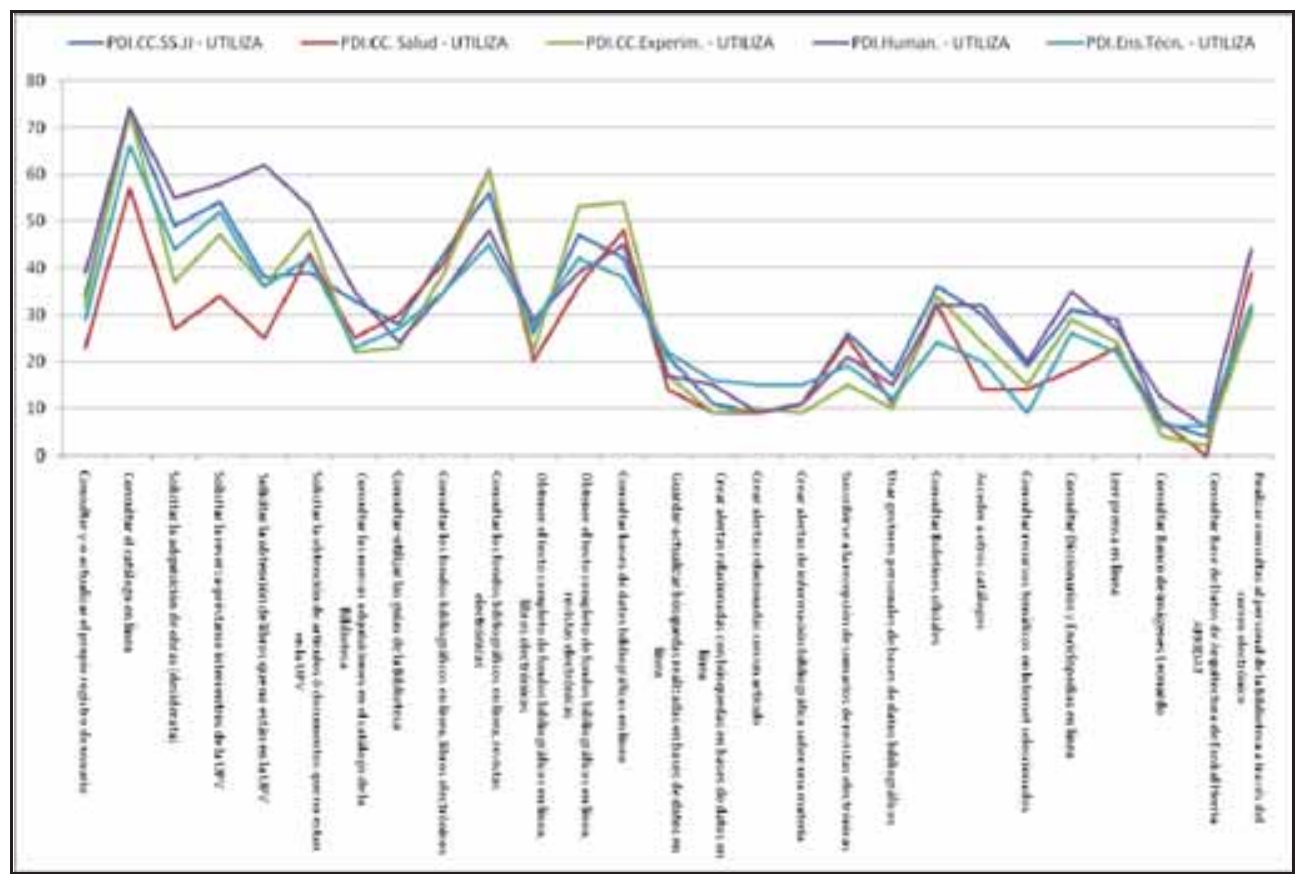

Gráfico 22: PDI. Utilización de servicios no presenciales por áreas.

En cuanto al uso por el PDI de los servicios no presenciales de la Biblioteca, el análisis muestra comportamientos dispares. Las diferencias más grandes entre áreas se producen en los servicios directamente relacionados con los fondos presentes

en la Biblioteca: solicitar la adquisición de obras, solicitar la reserva-préstamo intercentros, solicitar la obtención de libros que no están en la UPV. En ellos, el área de Humanidades es la que mayor uso hace y Ciencias de la Salud es el que menor uso hace.

En una visión general del resto de servicios, el área de Enseñanzas Técnicas es el que muestra una tendencia hacia los resultados más bajos, con la excepción de la creación de alertas en las que se muestra por encima del resto. Ciencias de la Salud muestra resultados paradójicos: el mayor uso de revistas electrónicas en línea (junto a Ciencias Experimentales) pero con la menor obtención de los textos completos de aquellas; alto porcentaje de uso de las bases de datos bibliográficas en línea pero de los menores en guardar-actualizar búsquedas y en la creación de alertas. Humanidades obtiene bajos resultados en los servicios relacionados con los recursos electrónicos, salvo en la obtención del texto completo de libros electrónicos en línea, pese a ser el que menos los consulta (junto con Enseñanzas Técnicas). Ciencias Experimentales hace un uso elevado de los fondos en línea. Ciencias Sociales y Jurídicas, por último, es el área que presenta el perfil más próximo al general, con porcentajes altos en el uso de fondos en línea y destacándose en el uso de gestores personales de bases de datos bibliográficos $\mathrm{y}$, lógicamente, en el de los boletines oficiales. 
Analizados los datos globalmente por áreas, el área de Ciencias Experimentales muestra, en general, un mayor grado de conocimiento de los servicios no presenciales de la Biblioteca. En el extremo contrario se sitúa el área de Enseñanzas Técnicas.

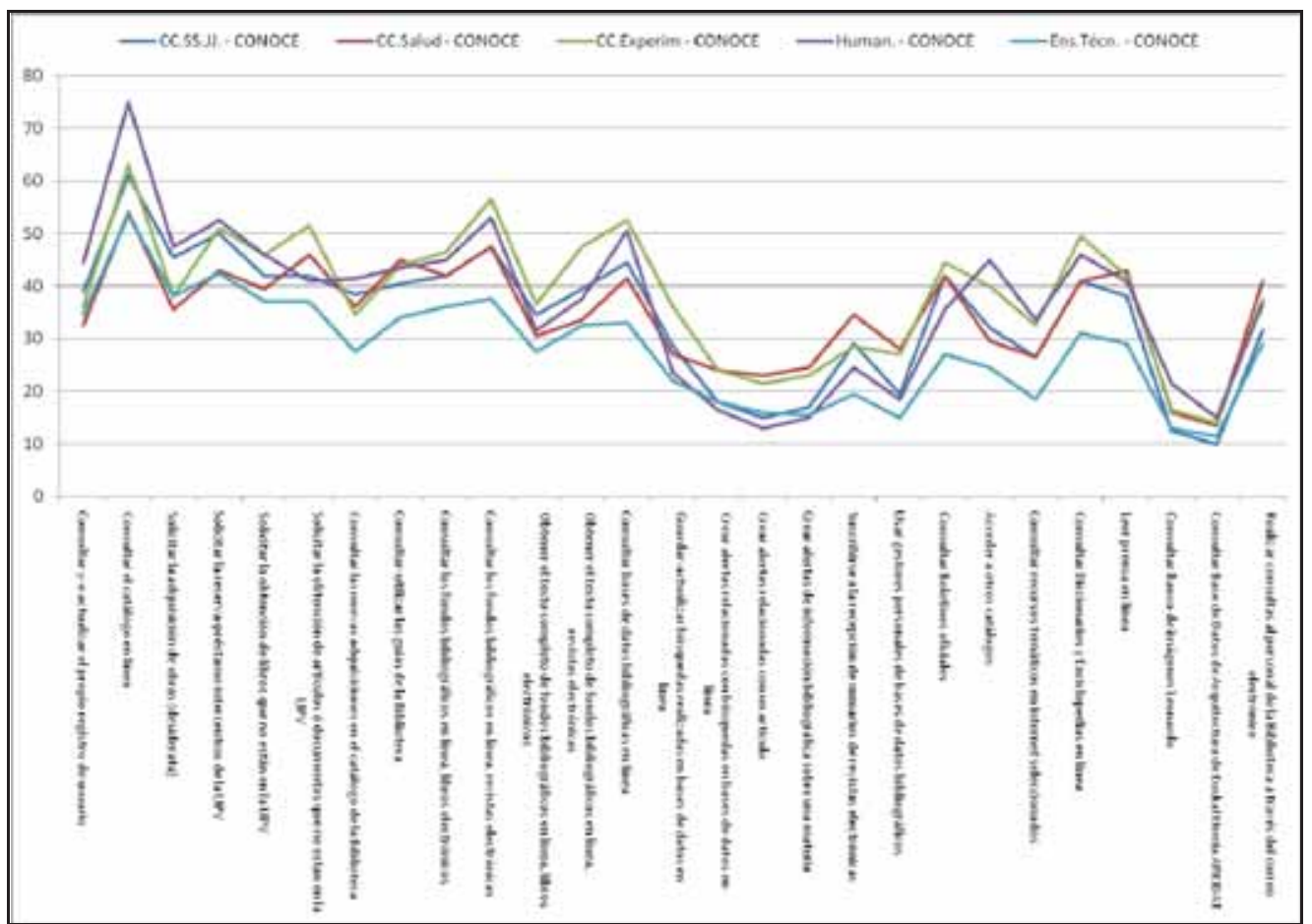

Gráfico 23: Conocimiento de servicios no presenciales por áreas.

Respecto al uso y de forma general, Humanidades muestra una trayectoria con niveles altos salvo en los servicios relacionados con guardar búsquedas y crear alertas. Ciencias Experimentales le sigue, superándole incluso en el uso de fondos en línea y en la obtención del texto completo de éstos. Ciencias Sociales y Jurídicas se mantiene en una zona intermedia con porcentajes altos en el uso de los gestores personales de bibliografía y en el uso de los boletines oficiales. Ciencias de la Salud muestra variaciones importantes con porcentajes bajos en los servicios más directamente relacionados con los materiales dispuestos en la Biblioteca y altos en algunos de los relacionados con los recursos electrónicos. Por último, el área de Enseñanzas Técnicas muestra una tendencia hacia porcentajes bajos salvo en los servicios más directamente relacionados con los materiales dispuestos en la Biblioteca y en guardar- actualizar búsquedas en bases de datos en línea y crear los diferentes tipos de alertas. 


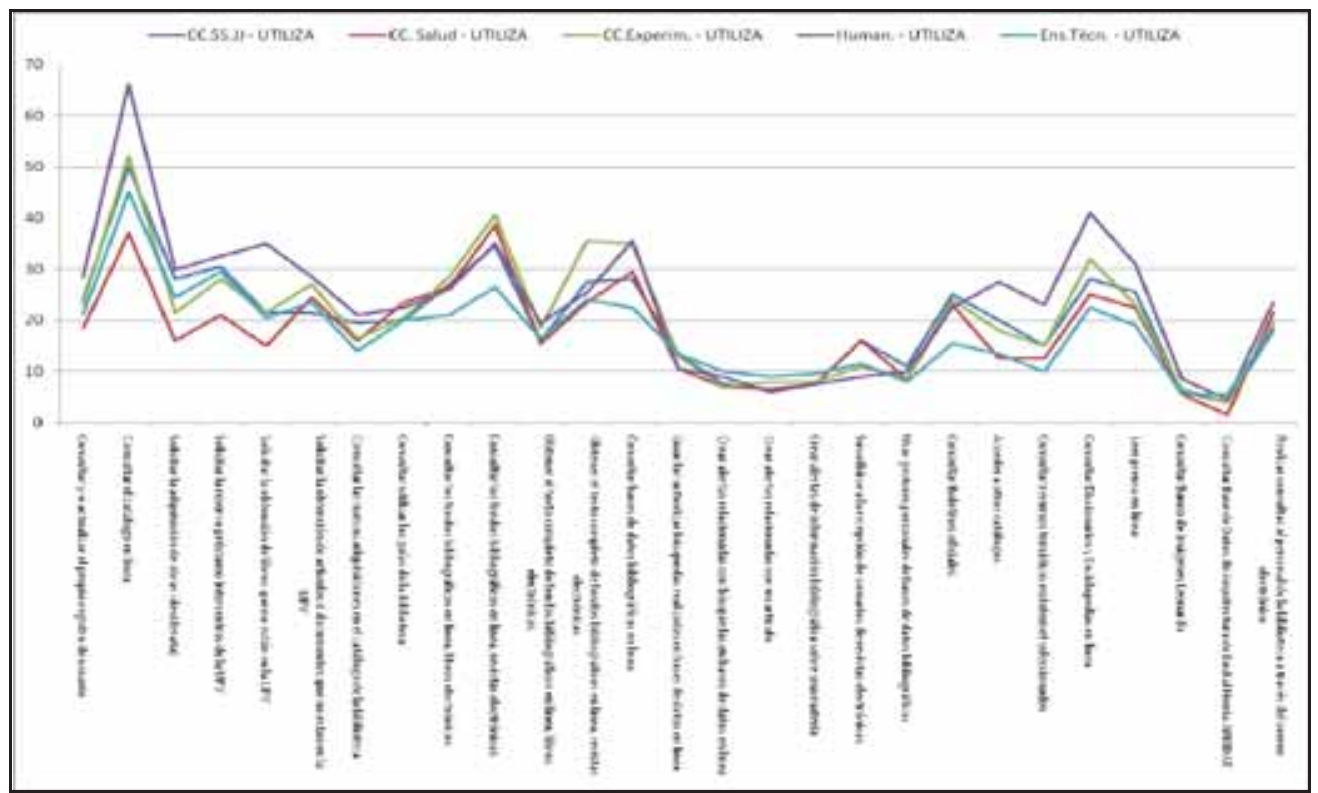

Gráfico 24: Utilización de servicios no presenciales por áreas.

\section{CONCLUSIONES}

Del análisis de los resultados obtenidos en este estudio se puede extraer las siguientes conclusiones:

- Se observa en el alumnado un desconocimiento importante de los servicios presenciales ofrecidos por la Biblioteca de la UPV/EHU.

- Este desconocimiento es aún más señalado en el caso de los servicios no presenciales y se hace necesario resaltar cómo ninguno de estos servicios es conocido por al menos la mitad del alumnado.

- Más de la mitad de los servicios no presenciales la Biblioteca de la UPV/EHU no es utilizado siquiera por una décima parte del alumnado.

- Se observan significativas variaciones entre el alumnado de las distintas áreas en el grado de conocimiento y uso de los diferentes servicios de la Biblioteca de la UPV/EHU, tanto en los presenciales como en los no presenciales.

- El grado de conocimiento y uso de los servicios de la Biblioteca de la UPV/EHU por parte del PDI es superior al del alumnado, tanto en los servicios presenciales como en los no presenciales.

- Entre el PDI de las distintas áreas, se detectan variaciones significativas en el grado de conocimiento y uso de los diferentes servicios de la Biblioteca de la UPV/EHU, tanto en el caso de los presenciales como en el de los no presenciales. 
- Contempladas las áreas globalmente, en el caso de los servicios presenciales el mayor grado de conocimiento y uso se registra en Humanidades. Las Enseñanzas Técnicas muestran en general un menor conocimiento y junto a Ciencias de la Salud los porcentajes de uso más bajos.

- En cuanto a los servicios no presenciales, el área de Ciencias Experimentales muestra un mayor conocimiento, mientras en el uso lo muestra el área de Humanidades. Los porcentajes más bajos de conocimiento los muestra el área de Enseñanzas Técnicas y en el uso los muestra de nuevo Enseñanzas Técnicas junto a Ciencias de la Salud.

\section{BIBLIOGRAFÍA}

ARIAS COELLO, Alicia; José SIMÓN MARTÍN. "Estudio de los hábitos de uso de los estudiantes en la Biblioteca de la Universidad Complutense de Madrid" [en línea]. Revista Española de Documentación Científica. V. 31, no 3 (2008). p. 413-431. (http://redc.revistas.csic.es/intex.php/redc/article/view/436/448) Consulta: 30-32010.

BAILIN, Alan; Ann GRAFSTEIN. "The evolution of academic libraries: the networked environment". The Journal of Academic Librarianship. V. 31, n. 4 (2005): 317-323.

BORREGO, Ángel [et al.]. "Use and users of electronic journal at catalan universities: the results of a survey" [en línea]. The Journal of Academic Librarianship, V. 33, n 1 (2007), p. 67-75.

(http://www.sciencedirect.com/science?_ob=MImg\&_imagekey=B6W504MKTXVT-1-

N\&_cdi $=6556 \&$ user $=984461 \&$ pii $=$ S0099133306001613\&_orig $=$ browse $\&$ cover Date $=01 \% 2 \mathrm{~F} 31 \% 2 \mathrm{~F} 2007 \&$ sk $=999669998 \& \mathrm{view}=\mathrm{c} \& \mathrm{wchp}=\mathrm{dGLbVlW}-$ zSkWA\&md5=bd2a2e2923aa8370c831741b20e29f7c\&ie=/sdarticle.pdf ) Consulta: 30-3-2010.

BRICALL, Josep M. (dir.).Informe Universidad 2000 [en línea]. Conferencia de Rectores de las Universidades Españolas, p. 460. (http://www.crue.org/informeuniv2000.htm). Consulta: 27-12-2007; (http://www.usal.es/ agptu/docs/InformeBricall/Bricallindice.html) Consulta: 2-42009.

BROWN, Sheridan; Alma SWAN. "Researchers' use of academic libraries and their services: a report commissioned by the Research Information Network and the Consortium of Research Libraries" [en línea]. Technical Paper. Research Information Network. (2007). (http://eprints.ecs.soton.ac.uk/13868/1/libraries-report-2007.pdf) Consulta: 30-3-2010.

FERNANDEZ MARTINEZ, Luis M ${ }^{\mathrm{a}}$. La Biblioteca de la Universidad del País Vasco ante la convergencia europea: conocimiento y uso de las TICS. Tesis inédita. Universidad de Zaragoza, Facultad de Filosofía y Letras, Departamento de Ciencias de la Documentación e Historia de la Ciencia, 2010. 
GARCÍA OCHOA, M ${ }^{\mathrm{a}}$ Luisa; Manuela CREGO CASTAÑO; Javier GIMENO PERELLÓ. Encuesta piloto a usuarios de la BUC. Biblioteca de la Facultad de CC. de la Información [en línea]. Documentos de trabajo UCM. Biblioteca, n. ${ }^{\circ}$ 93/9 (nov.1993). (http://www.ucm.es/BUCM/descargas/documento4518.pdf) Consulta: 30-3-2010.

GÓMEZ HERNÁNDEZ, José A. "Conocimiento, uso, valoración de los servicios y expectativas de los estudiantes universitarios de Murcia respecto de la Biblioteca universitaria". En: El bibliotecario ante la revolución tecnológica. Granada: Asociación Andaluza de Bibliotecarios, 1996. p. 185-206

HARREL, Charles B. The use of an academic library by university students. Ann Arbor, Michigan: UMI, 1988.

HERNÁNDEZ BLÁZQUEZ, Benjamín (dir.). Técnicas estadísticas de investigación social. Madrid: Díaz de Santos, 2001.

HILLER, Steve. "How different are they? A comparison by academic area of library use, priorities and information needs at the University of Washington" [en línea]. Issues in Science and Technology Librarianship. V. 33 (winter 2002). (http://www.istl.org/02-winter/article1.html) Consulta: 30-3-2010.

KENT, Allen [et al.]. Use of Library Materials: The University of Pittsburgh Study. New York: Marcel Dekker, 1979.

LAVIGNE, Richard de. Créditos ECTS y métodos para su asignación. 2003 [en línea]. (http://www.aneca.es/modal_eval/docs/doc_conv_gral1.pdf) Consulta: 30-08-2006.

MARTELL, Charles. "The elusive user: changing use patterns in academic libraries 1995 to 2004" [en línea]. College \& Research Libraries, V. 68, n. 5 (2007), p. 435444. (http://crl.acrl.org/content/68/5/435.full.pdf+html) Consulta: 30-3-2010.

MARTELL, Charles. "The absent user: physical use of academic library collection and services continues to decline 1995-2006" [en línea]. The Journal of Academic Librarianship, V. 34, n. 5 (2008), p. 400-407. (http://www.sciencedirect.com/science?_ob=MImg\&_imagekey=B6W504T4WM01-1-

7\&_cdi $=6556 \&$ user $=984461 \&$ pii $=$ S0099133308001018\&_orig=browse\&_cover Date $=09 \% 2 \mathrm{~F} 30 \% 2 \mathrm{~F} 2008 \&$ sk $=999659994 \& \mathrm{view}=\mathrm{c} \& \mathrm{wchp}=\mathrm{dGLbVtz}-$ zSkzS\&md5=aa8f2fb6c2ec083819c92d7bf6bf2bca\&ie=/sdarticle.pdf) Consulta: 3003-2010.

PEREA VEGA, Gustavo. "La Biblioteca de la Universidade da Coruña. Estudio con los alumnos" [en línea]. Revista Española de Documentación Científica, Vol. 25 (2002), $\mathrm{n}^{\circ}$ 1. p. 29-48. (http://redc.revistas.csic.es/index.php/redc/article/view/85/146) Consulta: 30-03-2010.

MARTELL, Charles. "The elusive user: changing use patterns in academic libraries 1995 to 2004" [en línea]. College \& Research Libraries, V. 68, n. 5 (2007), p. 435444. (http://crl.acrl.org/content/68/5/435.full.pdf+html) Consulta: 30-3-2010.

ORERA ORERA, Luisa. "La biblioteca universitaria ante el nuevo modelo social y educativo". El profesional de la información, 2007, julio-agosto, v. 16, n. 4, pp. 329-337.

(http://www.metapress.com/content/ew12032177137715/fulltext.pdf) Consulta: 30-32010. 
RAMOS-SIMÓN, Luis Fernando. "Prácticas de las bibliotecas universitarias argentinas. Reflexiones críticas en el contexto de la comunicación académica y sus representaciones en la Web (Mercedes Patalano)" [en línea]. El profesional de la información, 2009, septiembre-octubre, v. 18, n. 5, p. 570. (http://www.metapress.com/content/t2545474jh580558/fulltext.pdf) Consulta: 10-52010.

RODRIGUEZ BRAVO, Blanca. "Patterns of use of electronic journals in Spanish university libraries" [en línea]. Serials Review, V. 34, n. 2 (2008), p. 115-128. (http://www.sciencedirect.com/science?_ob=MImg\&_imagekey=B6W634S563W1-1-

$13 \&$ cdi $=6587 \&$ user $=984461 \&$ pii $=\mathrm{S} 0098791308000051 \&$ orig $=$ browse $\&$ cove rDate $=06 \% 2 \mathrm{~F} 30 \% 2 \mathrm{~F} 2008 \&$ sk $=999659997 \& \mathrm{view}=\mathrm{c} \& w \mathrm{wh}=-\mathrm{dGLzVtb}-$

zSkzS\&md5=3c5b8ed28e77441d7f009a764268fe9a5\&ie=/sdarticle.pdf) Consulta: 30-3-2010.

SRIDHAR, M.S. User research: a review of information behaviour studies in science and technology [en línea]. Bangalore: Biblio Infon Service, 1988. p. 17. (http://www.archive.org/details/UserResearchAReviewOfInformationBehaviuorStu diesInScienceAnd) Consulta: 30-3-2010.

SUAREZ BALSEIRO, Carlos [et.el.]. "Análisis de uso de las bases de datos de la biblioteca de la Universidad Carlos III de Madrid" [en línea]. Revista Española de Documentación Científica, V. 24, $\mathrm{n}^{\mathrm{o}} 1$ (2001), p. 23-35. (http://redc.revistas.csic.es/intex.php/redc/article/view/31/92) Consulta: 30-3-2010.

TENOPIR, Carol. Use and Users of Electronic Library Resources: An Overview and Analysis of Recent Research Studies [en línea].Washington, D.C.: Council on Library and Information Resources, 2003. (http://www.clir.org/pubs/reports/pub120/pub120.pdf) Consulta: 30-3-2010.

TROCHIM, Mary Kane; Arthur MILLER; William M.K. TROCHIM. Measuring the book circulation use of a small academic library collection: a manual. Washington, D.C.: Association of Research Libraries, 1985. 